Document downloaded from:

http://hdl.handle.net/10251/80839

This paper must be cited as:

Tur Valiente, M.; Albelda Vitoria, J.; Nadal Soriano, E.; Ródenas García, JJ. (2014). Imposing Dirichlet boundary conditions in hierarchical Cartesian meshes by means of stabilized Lagrange multipliers. International Journal for Numerical Methods in Engineering. 98(6):399-417. doi:10.1002/nme.4629.

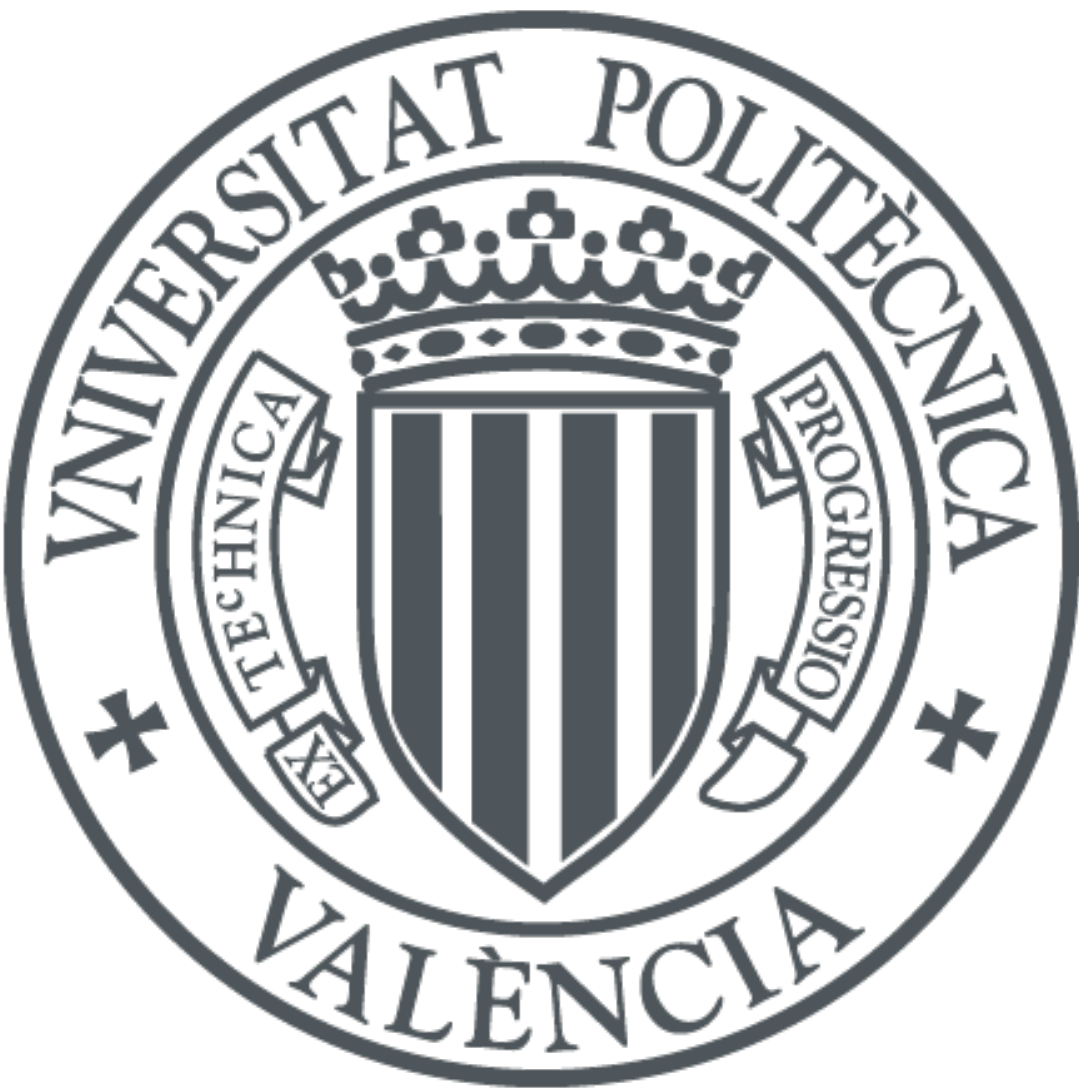

The final publication is available at

http://dx.doi.org/10.1002/nme.4629

Copyright Wiley

Additional Information

This is the pre-peer reviewed version of the following article: Tur, M., Albelda, J., Nadal, E. and Ródenas, J. J. (2014), Imposing Dirichlet boundary conditions in hierarchical Cartesian meshes by means of stabilized Lagrange multipliers. Int. J. Numer. Meth. Engng, 98: 399-417, which has been published in final form at http://dx.doi.org/10.1002/nme.4629 . This article may be used for non-commercial purposes in accordance with Wiley Terms and Conditions for Self-Archiving. 


\title{
Imposing Dirichlet boundary conditions in hierarchical Cartesian meshes by means of stabilized Lagrange multipliers
}

\author{
M. Tur ${ }^{1 *}$, J. Albelda ${ }^{1 *}$, E. Nadal ${ }^{1 *}$, J.J. Ródenas ${ }^{1 *}$ \\ 1: Centro de Investigación de Tecnología de Vehículos, Departamento de Ingeniería \\ Mecánica y de Materiales, Universitat Politècnica de Valencia, Camino de Vera s/n \\ 46022 Valencia (Spain)
}

\begin{abstract}
The use of Cartesian meshes independent of the geometry has some advantages over the traditional meshes used in the finite element method. The main advantage is that their use together with an appropriate hierarchical data structure reduces the computational cost of the Finite Element analysis. This improvement is based on the substitution of the traditional mesh generation process by an optimized procedure for intersecting the Cartesian mesh with the boundary of the domain and the use efficient solvers based on the hierarchical data structure. One major difficulty associated to the use of Cartesian grids is the fact that the mesh nodes do not, in general, lie over the boundary of the domain, increasing the difficulty to impose Dirichlet boundary conditions. In this paper, Dirichlet boundary conditions are imposed by means of the Lagrange multipliers technique. A new functional has been added to the initial formulation of the problem that has the effect of stabilizing the problem. The technique here presented allows for a simple definition of the Lagrange multipliers field, that even allow us to directly condense the degrees of freedom of the Lagrange multipliers at element level.
\end{abstract}

Keywords: Dirichlet boundary conditions, Lagrange multipliers, Stabilization, Immersed boundary method, Cartesian grid

\section{Introduction}

The finite element method (FEM) is one of the most widely used techniques for solving differential equations in the industrial and academic envi- 
ronments. The first step in the analysis process by means of the FEM is to discretize the geometry of the problem by subdividing the domain into subdomains of simple geometry called elements. The standard version of FEM is based on the use of a mesh of elements that conforms to the geometry of the domain to be analyzed. According to [1], a study at Sandia National Laboratories (USA) revealed that the generation of the finite element (FE) numerical model, including the process of creating a geometry suitable for analysis by the FEM and the subsequent process of meshing of the geometry, use $80 \%$ of the total time spent on the analysis, whereas only $20 \%$ is devoted to the numerical analysis which provides the solution of the problem. An alternative to reduce the time devoted to the generation of the model is to use an approach in which the FE mesh is independent of the geometry of the problem. In fact we can find numerous variants of the FEM which have followed this alternative to improve the performance of the method. Two of these techniques are the Extended Finite Element Method (XFEM) [2, 3] and the Generalized Finite Element Method (GFEM) [4, 5]. For example, in the XFEM, mainly devoted to the study of cracks in structural components, two types of enrichment functions are introduced. The first group of functions take into account the discontinuities of the displacement field into the elements cut by the crack, whereas the second type of functions describe the known behavior of the singular solution around the crack tip. The use of these enrichment functions avoids using fine meshes adapted to the crack geometry as in the standard FEM. In GFEM enrichment functions are also introduced to describe the known characteristics of the solution by means of the partition of unity method (PUM). The GFEM mesh can be independent of the geometry to be analyzed, such as in the 2D cartesian mesh used in the implementation GFEM III described in [4].

Another approach to improve the performance of the FEM is to use an auxiliary domain $\Omega_{E}$ containing the problem domain $\Omega$. In this case the domain discretized by FEM is $\Omega_{E}$ which is, in general, a domain with a simple geometry that can be easily meshed. Therefore this technique is closely related to the GFEM. In our implementation for 2D linear elasticity problems, $\Omega_{E}$ is a square whose discretization into quadrilateral elements is very simple. In the literature related to this subject, such techniques can be found under different names, such as Fictitious Domain [6, 7, 8, 27, 9], Implicit Meshing [10], Immersed Finite Element Method [11], Immersed Boundary Method [12, 13], Fixed Grid Finite Element Method [14, 15], and are described in [16] under the generalized term Finite elements in ambient 
space.

Since the mesh does not conform to the geometry it is necessary to use appropriate procedures for integration when evaluating the element matrices. In elements cut by the boundary of the domain, the integration must be only extended to the area of the element located inside of $\Omega$.

An important difference between this approach and the traditional FEM is the procedure to apply the Dirichlet boundary conditions since it must be taken into account that, in general, the FE nodes do not lie on the boundary. In this paper we propose to use the Lagrange multipliers method to impose Dirichlet boundary conditions. The use of this method in the case of the standard FEM was analyzed by [17] and [18]. The case of Cartesian meshes independent of the geometry is more complex to solve because the nodes of FE model are not located on the boundary. In this case it is more difficult to find compatible discretizations of displacements and multipliers that satisfy the InfSup condition [19]. Some procedures have been proposed to define the Lagrange multipliers that fulfill the InfSup in the case of linear elements in 2D [20] and 3D [21]. Another alternative, widely used in fluid dynamics $[22,23]$, is to use a procedure to stabilize the solution of the problem. Some methods to implement Dirichlet boundary conditions with stabilized solution in Cartesian meshes, can also be found in [24, 25, 26, 28, 27]. In this paper we propose a stabilization method suitable for $h$-refinement based on the use of hierarchical Cartesian grids where stabilization term does not depend on the solution of the current mesh. The definition of the Lagrange multipliers field allow us to directly condense the degrees of freedom of the Lagrange multipliers at element level.

\section{Problem statement}

Let $\Omega$ be a $2 \mathrm{D}$ bounded domain with a sufficiently smooth boundary $\Gamma$ where we want to find a displacement field $\mathbf{u}$ that satisfies the internal and boundary equilibrium equations for linear elasticity problems and the imposed displacements on the Dirichlet boundary. The boundary can be divided into non-overlapping parts $\Gamma_{\mathrm{D}}$ and $\Gamma_{\mathrm{N}}$, the Dirichlet and Neumann boundaries.

The weak form of the differential equation is expressed by the strain 
energy, which is defined as follows:

$$
\begin{aligned}
& a(\cdot, \cdot): \mathscr{V} \times \mathscr{V} \longrightarrow \mathbb{R} \\
& a(\mathbf{u}, \mathbf{v})=\int_{\Omega} \boldsymbol{\sigma}(\mathbf{u}): \boldsymbol{\epsilon}(\mathbf{v}) d \Omega
\end{aligned}
$$

where $\boldsymbol{\sigma}(\cdot)$ and $\boldsymbol{\epsilon}(\cdot)$ are the stress and strain tensors. The solution $\mathbf{u}$ is defined in the space $\mathscr{V}=\left(H^{1}(\Omega)\right)^{2}$.

We want to make use of the Lagrange multipliers technique to impose Dirichlet boundary conditions. The following integral can be defined to weakly impose these constraints

$$
\begin{aligned}
& b(\cdot, \cdot): \mathscr{M} \times \mathscr{V} \longrightarrow \mathbb{R} \\
& b(\boldsymbol{\mu}, \mathbf{u})=\int_{\Gamma_{\mathrm{D}}} \boldsymbol{\mu} \cdot \mathbf{u} d \Gamma
\end{aligned}
$$

where $\mathscr{M}=\left(H^{-\frac{1}{2}}\left(\Gamma_{\mathrm{D}}\right)\right)^{2}$ is the space where the Lagrange multipliers $\boldsymbol{\mu}$ are defined.

We define $\mathbf{f}_{v} \in L^{2}(\Omega)$ as the volumetric forces, $\mathbf{f}_{s} \in L^{2}\left(\Gamma_{\mathrm{N}}\right)$ as the surface loads and $\mathrm{g} \in \mathscr{M}^{\prime}$ the value of the displacements imposed on the boundary where $\mathscr{M}^{\prime}$ is the dual space of $\mathscr{M}$. The virtual work of the external forces can be defined as

$$
c(\mathbf{v})=\int_{\Omega} \mathbf{v} \cdot \mathbf{f}_{v} d \Omega+\int_{\Gamma_{\mathrm{N}}} \mathbf{v} \cdot \mathbf{f}_{s} d \Gamma
$$

The formulation of the problem can be written as follows:

Find $[\mathbf{u}, \boldsymbol{\lambda}] \in \mathscr{V} \times \mathscr{M}$ such that

$$
\begin{array}{ll}
a(\mathbf{u}, \mathbf{v})+b(\boldsymbol{\lambda}, \mathbf{v})=c(\mathbf{v}) & \forall \mathbf{v} \in \mathscr{V} \\
b(\boldsymbol{\mu}, \mathbf{u})=(\boldsymbol{\mu}, \mathbf{g}) & \forall \boldsymbol{\mu} \in \mathscr{M}
\end{array}
$$

where $(\cdot, \cdot)$ denotes the scalar product. The problem in equation (4) has a solution and this solution is unique $[29,19]$ provided that the spaces $\mathscr{V}$ and $\mathscr{M}$ are chosen so that the following conditions are satisfied:

(i) Continuity. The bilinear functionals are continuous, i.e., there exist two constants $C_{a}>0$ and $C_{b}>0$, such that

$$
\begin{array}{ll}
a(\mathbf{u}, \mathbf{v}) \leq C_{a}\|\mathbf{u}\|_{\mathscr{V}}\|\mathbf{v}\|_{\mathscr{V}} & \forall \mathbf{u}, \mathbf{v} \in \mathscr{V} \\
b(\boldsymbol{\mu}, \mathbf{v}) \leq C_{b}\|\boldsymbol{\mu}\|_{\mathscr{M}}\|\mathbf{v}\|_{\mathscr{V}} & \forall \boldsymbol{\mu} \in \mathscr{M}, \forall \mathbf{v} \in \mathscr{V}
\end{array}
$$


(ii) ElKer (Ellipticity in the Kernel). The functional $a(\cdot, \cdot)$ is coercive in the kernel of $b(\cdot, \cdot)$, i.e. there exists a constant $\alpha>0$ such that

$$
a(\mathbf{v}, \mathbf{v}) \geq \alpha\|\mathbf{v}\|_{\mathscr{V}}^{2}, \forall \mathbf{v} \neq 0 \text { satisfying } b(\boldsymbol{\lambda}, \mathbf{v})=0
$$

(iii) InfSup. The functional $b(\cdot, \cdot)$ has the following coercivity property: There exist a constant $\beta>0$ such that for $\boldsymbol{\mu} \neq 0$ and $\mathbf{v} \neq 0$ the following expression is satisfied

$$
\inf _{\boldsymbol{\mu} \in \mathscr{M}} \sup _{\mathbf{v} \in \mathscr{V}} \frac{b(\boldsymbol{\mu}, \mathbf{v})}{\|\boldsymbol{\mu}\|_{\mathscr{M}}\|\mathbf{v}\|_{\mathscr{V}}} \geq \beta
$$

The problem in (4) can be derived from a constrained minimization problem solved using the Lagrange multipliers method. The problem consist of finding the saddle point $[\mathbf{u}, \boldsymbol{\lambda}]$ (minimum respect to the variable and maximum respect to the multipliers) of the following functional:

$$
\mathscr{L}(\mathbf{v}, \boldsymbol{\mu})=\frac{1}{2} a(\mathbf{v}, \mathbf{v})+b(\boldsymbol{\mu}, \mathbf{v})-c(\mathbf{v})
$$

\subsection{Finite element discretization}

The problem in (4) is discretized using the finite element method, making use of the discretized spaces for the displacements $\mathscr{V}^{h} \subset \mathscr{V}$ and multipliers $\mathscr{M}^{h} \subset \mathscr{M}$. We have to solve the following problem

Find $\left[\mathbf{u}^{h}, \boldsymbol{\lambda}^{h}\right] \in \mathscr{V}^{h} \times \mathscr{M}^{h}$ such that

$$
\begin{array}{ll}
a\left(\mathbf{u}^{h}, \mathbf{v}^{h}\right)+b\left(\boldsymbol{\lambda}^{h}, \mathbf{v}^{h}\right)=c\left(\mathbf{v}^{h}\right) & \forall \mathbf{v}^{h} \in \mathscr{V}^{h} \\
b\left(\boldsymbol{\mu}^{h}, \mathbf{u}^{h}\right)=\left(\boldsymbol{\mu}^{h}, \mathbf{g}\right) & \forall \boldsymbol{\mu}^{h} \in \mathscr{M}^{h}
\end{array}
$$

The problem in (9) can be rewritten in a more compact form as follows:

Find $\left[\mathbf{u}^{h}, \boldsymbol{\lambda}^{h}\right] \in \mathscr{V}^{h} \times \mathscr{M}^{h}$ such that:

$Q\left(\left[\mathbf{u}^{h}, \boldsymbol{\lambda}^{h}\right],\left[\mathbf{v}^{h}, \boldsymbol{\mu}^{h}\right]\right)=F\left(\left[\mathbf{v}^{h}, \boldsymbol{\mu}^{h}\right]\right) \quad \forall\left[\mathbf{v}^{h}, \boldsymbol{\mu}^{h}\right] \in \mathscr{V}^{h} \times \mathscr{M}^{h}$

where

$$
\begin{aligned}
& Q\left(\left[\mathbf{u}^{h}, \boldsymbol{\lambda}^{h}\right],\left[\mathbf{v}^{h}, \boldsymbol{\mu}^{h}\right]\right)=a\left(\mathbf{u}^{h}, \mathbf{v}^{h}\right)+b\left(\boldsymbol{\lambda}^{h}, \mathbf{v}^{h}\right)+b\left(\boldsymbol{\mu}^{h}, \mathbf{u}^{h}\right) \\
& F\left(\left[\mathbf{v}^{h}, \boldsymbol{\mu}^{h}\right]\right)=c\left(\mathbf{v}^{h}\right)+\left(\boldsymbol{\mu}^{h}, \mathbf{g}\right)
\end{aligned}
$$


In this paper we are interested in imposing Dirichlet boundary conditions on a Finite Element model where the mesh is defined by a so-called Cartesian grid. The problem domain $\Omega$ is embedded in a regular grid of quadrilateral elements (Cartesian grid) independent of the boundary $\Gamma$ as shown in Figure (1). $H$-adaptive refinement is obtained by splitting each element into four new elements. Multi-point-constraints (MPC) are used to impose $C^{0}$ continuity between adjacent elements of different refinement level [30]. A maximum refinement difference of one level is allowed between adjacent elements.

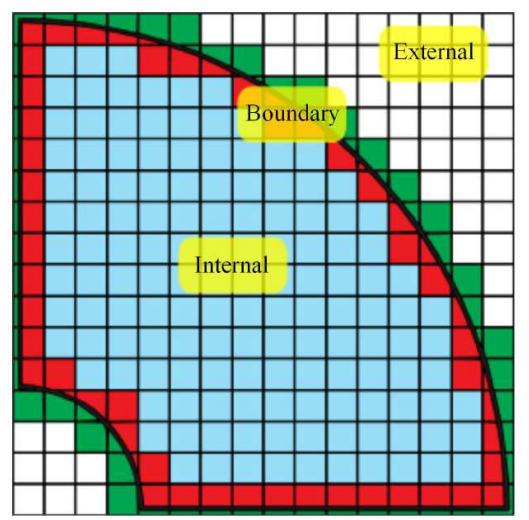

Figure 1: Cartesian mesh intersected by the geometry. We can observe the internal elements (blue), external elements (white) and boundary elements (red inside and green outside).

Standard isoparamentric four-node bilinear elements $\left(\mathcal{Q}_{4}\right)$ and eight-node quadratic elements $\left(\mathcal{Q}_{8}\right)$ are considered for the interpolation of the displacement field into each element. Now we have to define the interpolation of the Lagrange multipliers. To do this while maintaining the theoretical convergence rate of the finite element method, we must find a space of Lagrange multipliers satisfying the stability conditions. The formulation in equation (10) is stable if the continuity, ElKer and InfSup conditions (equations (5), (6) and (7), respectively) are satisfied in the selected finite element space $\mathscr{V}^{h} \times \mathscr{M}^{h}$. The first two conditions are easily satisfied by almost any standard finite element discretization. The third condition InfSup can be easily satisfied for a given mesh, but the optimal convergence rate is only achieved if this condition is satisfied for a sequence of refined meshes with constant $\beta$ independent of the mesh size. This requires certain degree of compatibility 
between the displacement space and the space of Lagrange multipliers which is difficult to verify. The Vital Vertex method solves this problem for linear elements in 2D [20] and for linear tetrahedral elements in 3D [21].

The following mesh-dependent norms can be defined for the finite element spaces of displacements and Lagrange multipliers [18, 31]:

$$
\begin{aligned}
\left\|\mathbf{u}^{h}\right\|_{\mathscr{V}^{h}}^{2} & =\left|\mathbf{u}^{h}\right|_{H^{1}, \Omega}^{2}+\sum_{e} h_{e}^{-1}\left\|\mathbf{u}^{h}\right\|_{L^{2}, \Gamma_{\mathrm{D}}^{e}}^{e} \\
\left\|\boldsymbol{\lambda}^{h}\right\|_{\mathscr{M}^{h}}^{2} & =\sum_{e} h_{e}\left\|\boldsymbol{\lambda}^{h}\right\|_{L^{2}, \Gamma_{\mathrm{D}}^{e}}^{2}
\end{aligned}
$$

where the summation extends to all elements of the mesh that are intersected by $\Gamma_{\mathrm{D}}$ and $h_{e}$ is the size of the contour segment corresponding to each element.

\section{Stabilization method}

In this work we are interested in freely defining the Lagrange multiplier field. Therefore the InfSup condition will not be verified and we need other method to obtain the optimal convergence. As pointed out above, an alternative to solve the problem in (4) are the so-called stabilization methods. These methods provide more flexibility in the selection of the Lagrange multipliers space $\mathscr{M}^{h}$, since they do not require to satisfy the InfSup condition. The strategy consists in modifying (4) (or equivalently modifying the Lagrangian in (8)), by adding a function that stabilizes the whole problem (10). Stabilization methods can be classified into two groups: residual-based methods and projection methods.

In general terms, residual-based methods are obtained by adding two terms to the mixed problem (the differential equation and the constraint equation) weighted by certain functions. Different methods are obtained depending on the selection of the weighting functions providing different stability and convergence properties of the stabilized problem. In [22] Barbosa and Hughes describe different residual methods proposed in the context of fluid dynamics. The advantage of these methods is that they stabilize the solution while maintaining consistency. The drawback is that the optimal convergence often depends on certain parameters that must be defined by the user. The projection methods are not based on the residual and, in general, they are not consistent in the sense that the exact solution of the continuous problem

(4) does not satisfy the equation of the discretized stabilized one (9). 
In general, these methods can be written by adding a new stabilization term to the discretization of the Lagrangian of equation (8) which can be written as:

$$
\begin{aligned}
& \mathscr{L}_{S}\left(\mathbf{v}^{h}, \boldsymbol{\mu}^{h}\right)=\frac{1}{2} a\left(\mathbf{v}^{h}, \mathbf{v}^{h}\right)-c\left(\mathbf{v}^{h}\right)+b\left(\boldsymbol{\mu}^{h}, \mathbf{v}^{h}\right)-\frac{1}{2} s\left(\boldsymbol{\mu}^{h}-\boldsymbol{T}, \boldsymbol{\mu}^{h}-\boldsymbol{T}\right) \\
& \text { with } s\left(\boldsymbol{\phi}^{h}, \boldsymbol{\theta}^{h}\right)=k \sum_{e} h_{e} \int_{\Gamma_{\mathrm{D}}^{e}} \boldsymbol{\phi}^{h} \cdot \boldsymbol{\theta}^{h} d \Gamma
\end{aligned}
$$

where $h_{e}$ is the size of the Dirichlet boundary corresponding to each element and $k$ is a positive penalty parameter that should not affect the convergence rate of the method. The different stabilization methods are obtained by setting the value of the term $\boldsymbol{T}$. The idea behind these methods is that the choice of the Lagrange multipliers interpolation imposes too many constraints on the degrees of freedom of the problem. An over-constrained problem implies that the constraint equations tend to become increasingly dependent and, therefore, the value of the multiplier tend to become less bounded. The stabilization term prevents excessive oscillations of the Lagrange multipliers solution, since it penalizes the difference between the multipliers and a given function $\boldsymbol{T}$. In practice, an adequately defined function $\boldsymbol{T}$ will properly couple the multipliers eliminating redundant constraint equations, thus recovering the optimal convergence rate of the method.

In [23] Bochev et al. propose a polynomial projection for the Darcy problem. Polynomial interpolations of the same degree are defined both, for the multipliers and for variable $\mathbf{u}$. The term $\boldsymbol{T}$ is then calculated as the projection of the Lagrange multipliers field in a polynomial space, one degree lower than that used for variable $\mathbf{u}$, defined element-wise. This will penalize the higher degree terms in the Lagrange multipliers field while maintaining the low degree terms required to achieve optimal convergence. Reference [32] demonstrates that if the term $\boldsymbol{T}$ corresponds to a discretization that fulfills the InfSup condition, then the Lagrange multipliers field does not need to satisfy this requirement to achieve the optimal convergence rate with the stabilized problem. Based on this property several functions for the stabilization term can be defined.

The Barbosa-Hughes stabilization [33] was used to impose Dirichlet boundary conditions in the standard finite element method. In this case the term $\boldsymbol{T}$ in (12) would correspond to the traction vector $\boldsymbol{T}=-\boldsymbol{\sigma}\left(\mathbf{u}^{\boldsymbol{h}}\right) \cdot \mathbf{n}$. Sten- 
berg [31] demonstrated the the Barbosa-Hughes stabilization was equivalent to the classical Nitsche's method. In the context of immersed boundary, the Nitsche's method was analyzed in [24, 28] and its stabilized version in [25]. Haslinger and Renard [25] pointed out that the use of Barbosa-Hughes stabilization to immersed boundary method needs additional regularity assumption on the intersection of the mesh with the boundary. To overcome the problem they proposed a technique which consists in using an extrapolation operator that takes into account the internal degrees of freedom for small intersected elements. This idea was also explored by Codina and Baiges [34].

\subsection{Stabilization term for Cartesian grids}

In this paper we are interested in $h$-refinement analysis techniques based on the use of Cartesian grids where the final solution is evaluated by generating a sequence increasingly refined finite element meshes obtained by element splitting. This procedure has some advantages as described in [35, 30] mainly the reduction of the computational cost of the $\mathrm{FE}$ analysis. In this case it makes sense to propose a stabilization term based on the solution obtained with the previous mesh of the sequence of meshes used for the analysis. We propose to define the term $\boldsymbol{T}$ for mesh $i$ as the solution of the boundary tractions in the previous mesh $\boldsymbol{T}^{*}=-\boldsymbol{\sigma}\left(\mathbf{u}_{\boldsymbol{i - 1}}^{h}\right) \cdot \mathbf{n}$, where $\mathbf{n}$ is the vector normal to the Dirichlet boundary and $\mathbf{u}_{i-1}^{h}$ are the displacements evaluated in mesh $i-1$. Unlike other methods in the literature, in this case, the additional term is independent of the field variables. Therefore it does not introduce any new matrices in the finite element discretization that must be evaluated and assembled. Furthermore, observing (12) we realize that, since in the exact solution the Lagrange multipliers field is equal to the tractions along the Dirichlet boundary, the stabilization term becomes increasingly smaller. Therefore the method is consistent in the limit when the element size tends to zero.

Thus, from equation (12), the stabilized problem can be formulated as follows:

Find $\left[\mathbf{u}^{h}, \boldsymbol{\lambda}^{h}\right] \in \mathscr{V}^{h} \times \mathscr{M}^{h}$ such that:

$Q_{S}\left(\left[\mathbf{u}^{h}, \boldsymbol{\lambda}^{h}\right],\left[\mathbf{v}^{h}, \boldsymbol{\mu}^{h}\right]\right)=F_{S}\left(\left[\mathbf{v}^{h}, \boldsymbol{\mu}^{h}\right]\right) \quad \forall\left[\mathbf{v}^{h}, \boldsymbol{\mu}^{h}\right] \in \mathscr{V}^{h} \times \mathscr{M}^{h}$

where

$$
\begin{aligned}
& Q_{S}\left(\left[\mathbf{u}^{h}, \boldsymbol{\lambda}^{h}\right],\left[\mathbf{v}^{h}, \boldsymbol{\mu}^{h}\right]\right)=a\left(\mathbf{u}^{h}, \mathbf{v}^{h}\right)+b\left(\boldsymbol{\lambda}^{h}, \mathbf{v}^{h}\right)+b\left(\boldsymbol{\mu}^{h}, \mathbf{u}^{h}\right)-s\left(\boldsymbol{\mu}^{h}, \boldsymbol{\lambda}^{h}\right) \\
& F_{S}\left(\left[\mathbf{v}^{h}, \boldsymbol{\mu}^{h}\right]\right)=\left(\mathbf{f}, \mathbf{v}^{h}\right)+\left(\boldsymbol{\mu}^{h}, \mathbf{g}\right)-s\left(\boldsymbol{\mu}^{h}, \boldsymbol{T}^{*}\right)
\end{aligned}
$$


The bilinear form $Q_{S}(\cdot, \cdot)$ is continuous and the following norm can be defined

$$
\|\|\left[\mathbf{u}^{h}, \boldsymbol{\lambda}^{h}\right]\left\|\left.\right|^{2}=\right\| \mathbf{u}^{h}\left\|_{\mathscr{V} h}^{2}+\right\| \boldsymbol{\lambda}^{h} \|_{\mathscr{M}^{h}}^{2}
$$

As we shall see later, the problem in equation (13) is stable. To ensure that the stabilized problem is well constrained, we follow the approach proposed in [31] and used also in [32]. We will assume that for each $\mathbf{u}^{h} \in \mathscr{V}^{h}$ there exists a continuous projection $\xi^{h}\left(\mathbf{u}^{h}\right) \in \mathscr{M}^{h}$ that, as discussed below, ensure that the problem is well constrained and prevent rigid body motions. The following properties are required:

$$
\begin{aligned}
& \text { (i) }\left\|\xi^{h}\left(\mathbf{u}^{h}\right)\right\|_{\mathscr{M}^{h}} \leq C_{\xi}\left\|\mathbf{u}^{h}\right\|_{\mathscr{V} h} \\
& \text { (ii) } \alpha_{\xi}\left\|\mathbf{u}^{h}\right\|_{\mathscr{V} h}^{2} \leq a\left(\mathbf{u}^{h}, \mathbf{u}^{h}\right)+b\left(\xi^{h}\left(\mathbf{u}^{h}\right), \mathbf{u}^{h}\right)
\end{aligned}
$$

with $C_{\xi}$ and $\alpha_{\xi}$ positive constants.

The stability of the problem in (13) is demonstrated in the following theorem:

Theorem 1. Given $\left[\mathbf{u}^{h}, \boldsymbol{\lambda}^{h}\right] \in \mathscr{V}^{h} \times \mathscr{M}^{h}$ satisfying the properties in (15), there exists a positive constant $\beta>0$ independent of the mesh size $h$ such that the following expression is satisfied:

$$
\sup _{\left[\mathbf{v}^{h}, \boldsymbol{\mu}^{h}\right] \in \mathscr{V} h \times \mathscr{M}^{h}} \frac{Q_{S}\left(\left[\mathbf{u}^{h}, \boldsymbol{\lambda}^{h}\right],\left[\mathbf{v}^{h}, \boldsymbol{\mu}^{h}\right]\right)}{\left|\left\|\left[\mathbf{v}^{h}, \boldsymbol{\mu}^{h}\right]\right\|\right|} \geq \beta\left|\left\|\left[\mathbf{u}^{h}, \boldsymbol{\lambda}^{h}\right]\right\|\right| \forall\left[\mathbf{u}^{h}, \boldsymbol{\lambda}^{h}\right] \in \mathscr{V}^{h} \times \mathscr{M}^{h}
$$

\section{Proof:}

For any variable $\left[\mathbf{u}^{h}, \boldsymbol{\lambda}^{h}\right]$, it will be sufficient to show that the inequality holds for a certain value of $\left[\mathbf{v}^{h}, \boldsymbol{\mu}^{h}\right]$, since the supremum is always greater than or equal to a particular value. Taking $\mathbf{v}^{h}=\mathbf{u}^{h}$ and $\boldsymbol{\mu}^{h}=-\left(\boldsymbol{\lambda}^{h}-\xi^{h}\left(\mathbf{u}^{h}\right)\right)$, where $\xi^{h}\left(\mathbf{u}^{h}\right)$ satisfies the properties in (15), we have

$Q_{S}\left(\left[\mathbf{u}^{h}, \boldsymbol{\lambda}^{h}\right],\left[\mathbf{v}^{h}, \boldsymbol{\mu}^{h}\right]\right)=Q_{S}\left(\left[\mathbf{u}^{h}, \boldsymbol{\lambda}^{h}\right],\left[\mathbf{u}^{h},-\left(\boldsymbol{\lambda}^{h}-\xi^{h}\left(\mathbf{u}^{h}\right)\right)\right]\right)=$ $a\left(\mathbf{u}^{h}, \mathbf{u}^{h}\right)+b\left(\boldsymbol{\lambda}^{h}, \mathbf{u}^{h}\right)+b\left(-\left(\boldsymbol{\lambda}^{h}-\xi^{h}\left(\mathbf{u}^{h}\right)\right), \mathbf{u}^{h}\right)-s\left(-\left(\boldsymbol{\lambda}^{h}-\xi^{h}\left(\mathbf{u}^{h}\right)\right), \boldsymbol{\lambda}^{h}\right)=$ $a\left(\mathbf{u}^{h}, \mathbf{u}^{h}\right)+b\left(\xi^{h}\left(\mathbf{u}^{h}\right), \mathbf{u}^{h}\right)+s\left(\left(\boldsymbol{\lambda}^{h}-\xi^{h}\left(\mathbf{u}^{h}\right)\right), \boldsymbol{\lambda}^{h}\right)$

Applying expression (ii) from equation (15) and taking into account the definition of the stabilization term in (12) and the norm of the multiplier in 
(11) together with expression $(i)$ in (15), we obtain

$$
\begin{aligned}
& Q_{S}\left(\left[\mathbf{u}^{h}, \boldsymbol{\lambda}^{h}\right],\left[\mathbf{v}^{h}, \boldsymbol{\mu}^{h}\right]\right) \geq \\
& \alpha_{\xi}\left\|\mathbf{u}^{h}\right\|_{\mathscr{V} h}^{2}+\frac{1}{2} s\left(\boldsymbol{\lambda}^{h}, \boldsymbol{\lambda}^{h}\right)-\frac{1}{2} s\left(\xi^{h}\left(\mathbf{u}^{h}\right), \xi^{h}\left(\mathbf{u}^{h}\right)\right)+\frac{1}{2} s\left(\boldsymbol{\lambda}^{h}-\xi^{h}\left(\mathbf{u}^{h}\right), \boldsymbol{\lambda}^{h}-\xi^{h}\left(\mathbf{u}^{h}\right)\right) \geq \\
& \alpha_{\xi}\left\|\mathbf{u}^{h}\right\|_{\mathscr{V} h}^{2}+\frac{k}{2} \sum_{e} h_{e}\left\|\boldsymbol{\lambda}^{h}\right\|_{L^{2}, \Gamma_{\mathrm{D}}^{e}}^{e}-\frac{k C_{\xi}}{2}\left\|\mathbf{u}^{h}\right\|_{\mathscr{V} h}^{2}+\underbrace{\frac{1}{2} s\left(\boldsymbol{\lambda}^{h}-\xi^{h}\left(\mathbf{u}^{h}\right), \boldsymbol{\lambda}^{h}-\xi^{h}\left(\mathbf{u}^{h}\right)\right)}_{\geq 0} \geq \\
& \left(\alpha_{\xi}-\frac{k C_{\xi}}{2}\right)\left\|\mathbf{u}^{h}\right\|_{\mathscr{V} h}^{2}+\frac{k}{2}\left\|\boldsymbol{\lambda}^{h}\right\|_{\mathscr{M}^{h}}^{2}
\end{aligned}
$$

Thus, for a sufficiently small value of the stabilization parameter $k$, a positive constant $\beta$ independent of the mesh size can be defined such that the following expression is satisfied

$$
Q_{S}\left(\left[\mathbf{u}^{h}, \boldsymbol{\lambda}^{h}\right],\left[\mathbf{v}^{h}, \boldsymbol{\mu}^{h}\right]\right) \geq \beta \sqrt{1+C_{\xi}^{2}}\left(\left\|\mathbf{u}^{h}\right\|_{\mathscr{V}^{h}}^{2}+\left\|\boldsymbol{\lambda}^{h}\right\|_{\mathscr{M}^{h}}^{2}\right)
$$

On the other hand, recalling the definition of $\mathbf{v}^{h}$ and $\boldsymbol{\mu}^{h}$ above and taking into account (15) (i), the following expression is satisfied

$$
\begin{aligned}
\left\|\mathbf{v}^{h}\right\|_{\mathscr{V}^{h}}^{2}+\left\|\boldsymbol{\mu}^{h}\right\|_{\mathscr{M}^{h}}^{2}=\left\|\mathbf{u}^{h}\right\|_{\mathscr{V}^{h}}^{2}+ & \left\|\boldsymbol{\lambda}^{h}-\xi^{h}\left(\mathbf{u}^{h}\right)\right\|_{\mathscr{M}^{h}}^{2} \leq \\
& \left(1+C_{\xi}^{2}\right)\left(\left\|\mathbf{u}^{h}\right\|_{\mathscr{V}^{h}}^{2}+\left\|\boldsymbol{\lambda}^{h}\right\|_{\mathscr{M}^{h}}^{2}\right)
\end{aligned}
$$

thus verifying the theorem.

\section{A priori error estimates}

Once the stability of the problem has been proven it is easy to show that the stabilized problem can be solved and has a unique solution. The optimal convergence rate of the method, still to be proven, is analyzed in this section. The a priori error estimate is based on the following theorem:

Theorem 2. Let $[\mathbf{u}, \boldsymbol{\lambda}] \in \mathscr{V} \times \mathscr{M}$ be the solution of the continuous problem (4) and $\left[\mathbf{u}^{h}, \boldsymbol{\lambda}^{h}\right] \in \mathscr{V}^{h} \times \mathscr{M}^{h}$ the solution of the discrete stabilized problem of equation (13) that fulfills equation (15). The following expression is satisfied:

$$
\begin{aligned}
& \left\|\mathbf{u}-\mathbf{u}^{h}\right\|_{\mathscr{V} h}+\left\|\boldsymbol{\lambda}-\boldsymbol{\lambda}^{h}\right\|_{\mathscr{M}^{h}} \leq \\
& \quad C\left(\inf _{\mathbf{v}^{h \in \mathscr{V} h}}\left\|\mathbf{u}-\mathbf{v}^{h}\right\|_{\mathscr{V} h}+\inf _{\boldsymbol{\mu}^{h} \in \mathscr{M}^{h}}\left\|\boldsymbol{\lambda}-\boldsymbol{\mu}^{h}\right\|_{\mathscr{M}^{h}}+k\left\|\boldsymbol{\lambda}-\boldsymbol{T}^{*}\right\|_{\mathscr{M}^{h}}\right)
\end{aligned}
$$

where $C$ is a positive constant independent of the mesh size. 


\section{Proof:}

We follow the ideas presented in [23] but adapted to our stabilized functional. Any $[\mathbf{v}, \boldsymbol{\mu}] \in \mathscr{V} \times \mathscr{M}$ satisfies (4). As $\mathscr{V}^{h} \times \mathscr{M}^{h}$ is a subspace of $\mathscr{V} \times \mathscr{M}$, in particular, any $\left[\mathbf{v}^{h}, \boldsymbol{\mu}^{h}\right] \in \mathscr{V}^{h} \times \mathscr{M}^{h}$ will also satisfy (4). Therefore we can write

$$
\begin{aligned}
a\left(\mathbf{u}, \mathbf{v}^{h}\right)+b\left(\boldsymbol{\lambda}, \mathbf{v}^{h}\right) & =\left(\mathbf{f}, \mathbf{v}^{h}\right) & & \forall \mathbf{v}^{h} \in \mathscr{V}^{h} \\
b\left(\boldsymbol{\mu}^{h}, \mathbf{u}\right) & =\left(\boldsymbol{\mu}^{h}, \mathbf{g}\right) & & \forall \boldsymbol{\mu}^{h} \in \mathscr{M}^{h}
\end{aligned}
$$

Including the term $-s\left(\boldsymbol{\mu}^{h}, \boldsymbol{\lambda}\right)$ on both sides of the second equation of the above expression and subtracting equation (13) corresponding to the stabilized problem from the resulting expression, we have:

$$
\begin{array}{rlrl}
a\left(\mathbf{u}-\mathbf{u}^{h}, \mathbf{v}^{h}\right)+b\left(\boldsymbol{\lambda}-\boldsymbol{\lambda}^{h}, \mathbf{v}^{h}\right) & =0 \quad \forall \mathbf{v}^{h} \in \mathscr{V}^{h} \\
b\left(\boldsymbol{\mu}^{h}, \mathbf{u}-\mathbf{u}^{h}\right)-s\left(\boldsymbol{\mu}^{h}, \boldsymbol{\lambda}-\boldsymbol{\lambda}^{h}\right) & =-s\left(\boldsymbol{\mu}^{h}, \boldsymbol{\lambda}-\boldsymbol{T}^{*}\right) & \forall \boldsymbol{\mu}^{h} \in \mathscr{M}^{h}
\end{array}
$$

or, in compact form

$$
Q_{S}\left(\left[\mathbf{u}-\mathbf{u}^{h}, \boldsymbol{\lambda}-\boldsymbol{\lambda}^{h}\right],\left[\mathbf{v}^{h}, \boldsymbol{\mu}^{h}\right]\right)=-s\left(\boldsymbol{\mu}^{h}, \boldsymbol{\lambda}-\boldsymbol{T}^{*}\right)
$$

We consider now any $\left[\mathbf{w}^{h}, \boldsymbol{\nu}^{h}\right] \in \mathscr{V}^{h} \times \mathscr{M}^{h}$. We have that

$$
\begin{aligned}
\left\|\mathbf{u}-\mathbf{u}^{h}\right\|_{\mathscr{V}^{h}}+\left\|\boldsymbol{\lambda}-\boldsymbol{\lambda}^{h}\right\|_{\mathscr{M}^{h}} \leq \\
\left\|\mathbf{u}-\mathbf{w}^{h}\right\|_{\mathscr{V}^{h}}+\left\|\boldsymbol{\lambda}-\boldsymbol{\nu}^{h}\right\|_{\mathscr{M}^{h}}+\left\|\mathbf{u}^{h}-\mathbf{w}^{h}\right\|_{\mathscr{V}^{h}}+\left\|\boldsymbol{\lambda}^{h}-\boldsymbol{\nu}^{h}\right\|_{\mathscr{M}^{h}}
\end{aligned}
$$

so, to prove (18) it suffices to bound the last two terms in the right hand side of the previous expression. Considering the coercivity of the stabilized functional (equation (16)) and equation (19) and operating we have

$$
\begin{aligned}
& \left|\left\|\left[\mathbf{u}^{h}-\mathbf{w}^{h}, \boldsymbol{\lambda}^{h}-\boldsymbol{\nu}^{h}\right]\right\|\right| \leq \frac{Q_{S}\left(\left[\mathbf{u}^{h}-\mathbf{w}^{h}, \boldsymbol{\lambda}^{h}-\boldsymbol{\nu}^{h}\right],\left[\mathbf{v}^{h}, \boldsymbol{\mu}^{h}\right]\right)}{\left\|\left[\mathbf{v}^{h}, \boldsymbol{\mu}^{h}\right]\right\| \mid}= \\
& \frac{Q_{S}\left(\left[\mathbf{u}^{h}-\mathbf{u}, \boldsymbol{\lambda}^{h}-\boldsymbol{\lambda}\right],\left[\mathbf{v}^{h}, \boldsymbol{\mu}^{h}\right]\right)+Q_{S}\left(\left[\mathbf{u}-\mathbf{w}^{h}, \boldsymbol{\lambda}-\boldsymbol{\nu}^{h}\right],\left[\mathbf{v}^{h}, \boldsymbol{\mu}^{h}\right]\right)}{\left\|\left[\mathbf{v}^{h}, \boldsymbol{\mu}^{h}\right]\right\| \mid}= \\
& \frac{-s\left(\boldsymbol{\mu}^{h}, \boldsymbol{\lambda}-\boldsymbol{T}^{*}\right)+Q_{S}\left(\left[\mathbf{u}-\mathbf{w}^{h}, \boldsymbol{\lambda}-\boldsymbol{\nu}^{h}\right],\left[\mathbf{v}^{h}, \boldsymbol{\mu}^{h}\right]\right)}{\left\|\left[\mathbf{v}^{h}, \boldsymbol{\mu}^{h}\right]\right\|}
\end{aligned}
$$

In what follows the symbol $\lesssim$ is used to denote $\leq$ up to a positive constant independent of the mesh size. Taking into account the continuity of the 
functional of the stabilized problem, from the above expression we have

$$
\begin{aligned}
& \left\|\mathbf{u}^{h}-\mathbf{w}^{h}\right\|_{\mathscr{V} h}+\left\|\boldsymbol{\lambda}^{h}-\boldsymbol{\nu}^{h}\right\|_{\mathscr{M}^{h}} \lesssim\left|\left\|\left[\mathbf{u}^{h}-\mathbf{w}^{h}, \boldsymbol{\lambda}^{h}-\boldsymbol{\nu}^{h}\right]\right\|\right| \leq \\
& \frac{-s\left(\boldsymbol{\mu}^{h}, \boldsymbol{\lambda}-\boldsymbol{T}^{*}\right)}{\left|\left\|\left[\mathbf{v}^{h}, \boldsymbol{\mu}^{h}\right]\right\|\right|}+\frac{\left|\left\|\left[\mathbf{u}-\mathbf{w}^{h}, \boldsymbol{\lambda}-\boldsymbol{\nu}^{h}\right]\right\|\right|\left|\left\|\left[\mathbf{v}^{h}, \boldsymbol{\mu}^{h}\right]\right\|\right|}{\|\|\left[\mathbf{v}^{h}, \boldsymbol{\mu}^{h}\right] \| \mid} \leq \\
& \frac{-s\left(\boldsymbol{\mu}^{h}, \boldsymbol{\lambda}-\boldsymbol{T}^{*}\right)}{\left|\left\|\left[\mathbf{v}^{h}, \boldsymbol{\mu}^{h}\right]\right\|\right|}+\left\|\mathbf{u}-\mathbf{w}^{h}\right\|_{\mathscr{V} h}+\left\|\boldsymbol{\lambda}-\boldsymbol{\nu}^{h}\right\|_{\mathscr{M}^{h}}
\end{aligned}
$$

Then, assuming that $\left\|\boldsymbol{\mu}^{h}\right\|_{\mathscr{M}^{h}} \neq 0$, the proof is completed taking into account that the stabilization term can be bounded using the Cauchy-Schwartz inequality:

$$
\frac{-s\left(\boldsymbol{\mu}^{h}, \boldsymbol{\lambda}-\boldsymbol{T}^{*}\right)}{\sqrt{\left\|\mathbf{v}^{h}\right\|_{\mathscr{V}^{h}}^{2}+\left\|\boldsymbol{\mu}^{h}\right\|_{\mathscr{M}^{h}}^{2}}} \leq \frac{\left|s\left(\boldsymbol{\mu}^{h}, \boldsymbol{\lambda}-\boldsymbol{T}^{*}\right)\right|}{\left\|\boldsymbol{\mu}^{h}\right\|_{\mathscr{M}^{h}}} \leq k \frac{\left\|\boldsymbol{\mu}^{h}\right\|_{\mathscr{M}^{h}}\left\|\boldsymbol{T}^{*}-\boldsymbol{\lambda}\right\|_{\mathscr{M}^{h}}}{\left\|\boldsymbol{\mu}^{h}\right\|_{\mathscr{M}^{h}}}
$$

If the stabilization term $\left\|\boldsymbol{\lambda}-\boldsymbol{T}^{*}\right\|_{\mathscr{M}^{h}}$ decreases properly, the previous theorem implies that the finite element solution is the best approximation to the exact solution that can be obtained within the finite element space. In our case, this term is the error of the finite element tractions computed in the Dirichlet boundary $\Gamma_{\mathrm{D}}$ measured in the mesh dependent norm. Its convergence rate is $O\left(h^{p+\frac{1}{2}}\right)$, where $p$ is the degree of interpolation of the displacements. Therefore, if the finite element interpolation has good approximation properties to the exact solution, the convergence of the method is optimum. Reference [31] shows that if the solution is smooth enough, the convergence rates of the best approximation of the finite element space measured in the $\|\cdot\|_{\mathscr{V}^{h}}$ and $\|\cdot\|_{\mathscr{M}^{h}}$ norms, at least have a convergence of orders $O\left(h^{p}\right)$ and $O\left(h^{q+\frac{3}{2}}\right)$, where $q$ is the degree of the Lagrange multipliers. The assumed regularity is that $\mathbf{u} \in H^{p+1}(\Omega)$ and $\boldsymbol{\lambda} \in H^{q+1}\left(\Gamma_{\mathrm{D}}\right)$.

Another alternative for $\boldsymbol{T}$ would be to use improved tractions obtained by a superconvergent patch stress recovery process which is known to provide a higher convergence rate that the FE solution [36, 37], although this has not been explored in this work.

\section{Interpolation of the Lagrange multipliers}

There are multiple alternatives to define the interpolation of the Lagrange multipliers. The only conditions that must be satisfied are: i) the interpolation must have good approximation properties to the exact solution, and ii) 
the interpolation must verify equation (15). With polynomial interpolation it is easy to satisfy the first condition. The second is related to the minimum number of constraints necessary to prevent rigid body motions.

One of these alternatives consist of using the so called naive approach $[20,38]$ in which a continuous piecewise linear or quadratic interpolation is defined based on the intersection points of the boundary with the edges of the elements. Another alternative used in [23, 26] would be to define the multiplier interpolation in the trace space of the displacements $\left.\mathbf{u}^{h}\right|_{\Gamma}$ on the boundary.

In this paper we propose to use a different interpolation scheme which has some advantages over previously proposed schemes, like the possibility to eliminate the Lagrange multipliers before assembling the system. The intersection points of the boundary with the edges of the elements define a subdivision of the boundary into possibly curved segments (see figure (2)). We define $n_{g}$ quadrature points on each segment. The polynomial interpolation of the multipliers on each segment is based on the multipliers at the quadrature points. Therefore, the use of $n_{g}$ quadrature points at each segment define a piecewise discontinuous polynomial of degree $q=n_{g}-1$ on the boundary. We consider a quadrature rule with $n_{g}=2$ points for $\mathcal{Q}_{4}$ elements and $n_{g}=3$ for $\mathcal{Q}_{8}$ elements, namely the interpolation of multipliers inside the elements in the first case is linear $(p=1$ and $q=1)$ and quadratic in the second ( $p=2$ and $q=2$ ). Therefore according to equation (18), the error in energy norm of the displacements will converge with order 1 for $\mathcal{Q}_{4}$ and with order 2 for $\mathcal{Q}_{8}$ elements.

Note that as the quadrature points are used to define the Lagrange multipliers interpolation, it is not necessary to explicitly calculate the shape functions to evaluate the integrals. The number of quadrature points ensures that the integrals on the boundary are exactly computed for straight segments, using Gaussian quadrature. This is the reason to choose the proposed values of $q$. Even though the best approximation of the multipliers has a high convergence rate, $O\left(h^{q+\frac{3}{2}}\right)$, the global convergence is limited by the approximation of the displacement field $O\left(h^{p}\right)$.

Let us show now that the interpolation scheme selected satisfies the properties of equation (15). We follow an approach similar to that presented in [32]. The function $\xi^{h}\left(\mathbf{u}^{h}\right)$ can be defined as the projection of the trace of the displacements over the Lagrange multiplier space divided by the size of element $h_{e}$, i.e. $\xi^{h}\left(\mathbf{u}^{h}\right)=\frac{\delta}{h_{e}} \Pi^{h}\left(\mathbf{u}^{h}\right)$ with $\delta>0$, where the projection $\Pi^{h} \in \mathscr{M}^{h}$ 

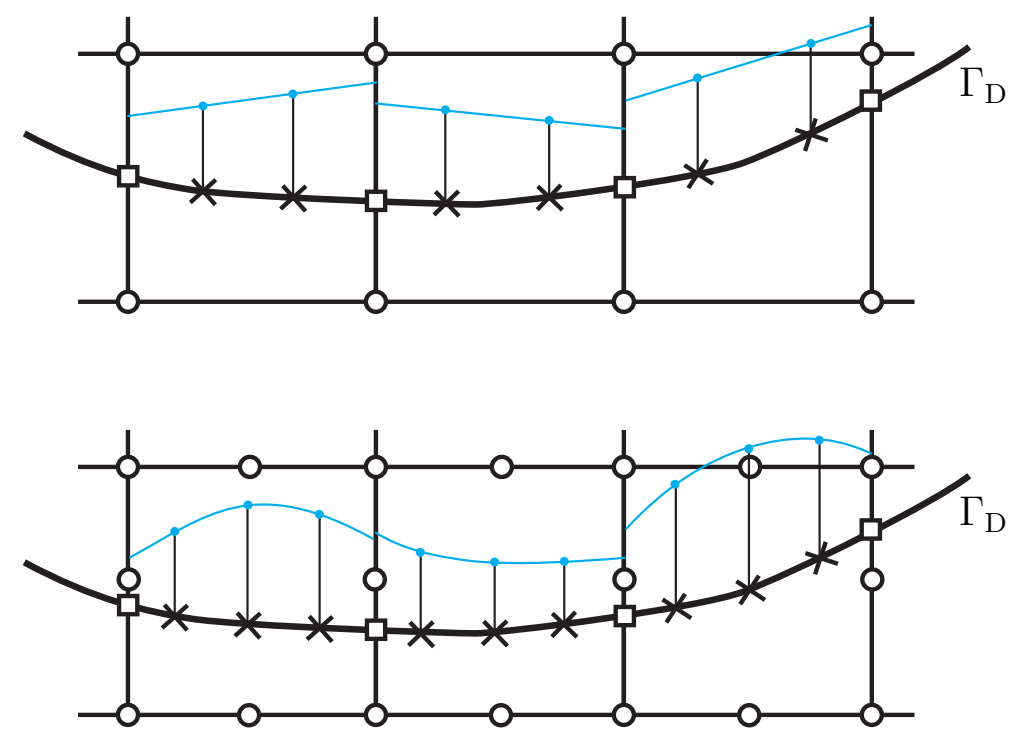

Figure 2: Examples of $\mathcal{Q}_{4}$ and $\mathcal{Q}_{8}$ meshes. Segmentation of the boundary $\Gamma_{\mathrm{D}}$ based on the intersection of the intersection of the boundary with the element edges (squares). The 'x' symbols denote the quadrature points where the Lagrange multipliers are used to define a piecewise discontinuous linear interpolation for $\mathcal{Q}_{4}$ elements and quadratic for $\mathcal{Q}_{8}$.

fulfills

$$
\int_{\Gamma_{\mathrm{D}}}\left(\Pi^{h}\left(\mathbf{u}^{h}\right)-\mathbf{u}^{h}\right) \cdot \boldsymbol{\mu}^{h} d \Gamma=0 \quad \forall \boldsymbol{\mu}^{h} \in \mathscr{M}^{h}
$$

Taking into account the definition of the norm in $\mathscr{M}^{h}$, and the continuity of the projection operator, it is obvious that the first equation in (15) is satisfied:

$$
\left\|\xi^{h}\left(\mathbf{u}^{h}\right)\right\|_{\mathscr{M}^{h}}^{2}=\delta \sum_{e} h_{e}^{-1}\left\|\Pi^{h}\left(\mathbf{u}^{h}\right)\right\|_{L^{2}, \Gamma_{\mathrm{D}}^{e}}^{2} \lesssim\left\|\mathbf{u}^{h}\right\|_{\mathscr{V}^{h}}^{2}
$$

To prove the second equation in (15) we need to bound the second term as follows

$$
\begin{aligned}
& b\left(\xi^{h}\left(\mathbf{u}^{h}\right), \mathbf{u}^{h}\right)=\sum_{e} \frac{\delta}{h_{e}} \int_{\Gamma_{\mathrm{D}}^{e}} \Pi^{h}\left(\mathbf{u}^{h}\right) \cdot \mathbf{u}^{h} d \Gamma= \\
& \sum_{e} \frac{\delta}{2} h_{e}^{-1}\left(\left\|\mathbf{u}^{h}\right\|_{L^{2}, \Gamma_{\mathrm{D}}^{e}}^{2}+\left\|\Pi^{h}\left(\mathbf{u}^{h}\right)\right\|_{L^{2}, \Gamma_{\mathrm{D}}^{e}}^{2}-\left\|\mathbf{u}^{h}-\Pi^{h}\left(\mathbf{u}^{h}\right)\right\|_{L^{2}, \Gamma_{\mathrm{D}}^{e}}^{2}\right)
\end{aligned}
$$

Then, taking into account the Poincaré inequality $h_{e}^{-1}\left\|\mathbf{u}^{h}-\Pi^{h}\left(\mathbf{u}^{h}\right)\right\|_{L^{2}, \Gamma_{\mathrm{D}}^{e}}^{2} \leq$ $C_{p}\left|\mathbf{u}^{h}\right|_{H^{1}, \Omega^{e}}^{2}$ that holds within each element and considering the equivalence 
between the energy norm and the seminorm $|\cdot|_{H^{1}, \Omega}$ (with positive constant $C_{d}$ ) we have:

$$
\begin{aligned}
& a\left(\mathbf{u}^{h}, \mathbf{u}^{h}\right)+b\left(\xi^{h}\left(\mathbf{u}^{h}\right), \mathbf{u}^{h}\right) \geq \\
& a\left(\mathbf{u}^{h}, \mathbf{u}^{h}\right)+\sum_{e} \frac{\delta}{2} h_{e}^{-1}\left(\left\|\mathbf{u}^{h}\right\|_{L^{2}, \Gamma_{\mathrm{D}}^{e}}^{2}-\left\|\mathbf{u}^{h}-\Pi^{h}\left(\mathbf{u}^{h}\right)\right\|_{L^{2}, \Gamma_{\mathrm{D}}^{e}}^{2}\right) \geq \\
& C_{d}\left|\mathbf{u}^{h}\right|_{H^{1}, \Omega}^{2}+\frac{\delta}{2} \sum_{e} h_{e}^{-1}\left\|\mathbf{u}^{h}\right\|_{L^{2}, \Gamma_{\mathrm{D}}^{e}}^{2}-\frac{\delta C_{p}}{2} \sum_{e}\left|\mathbf{u}^{h}\right|_{H^{1}, \Omega^{e}}^{2} \geq \alpha_{\xi}\left\|\mathbf{u}^{h}\right\|_{\mathscr{V}^{h}}^{2}
\end{aligned}
$$

\section{Implementation}

Standard shape functions are used to interpolate the displacement as $\mathbf{u}^{h}=\mathbf{N d}$, where $\mathbf{d}$ is the vector of nodal displacements. After evaluating the finite element discretization, the stabilized problem of equation (13) can be obtained by assembling the contribution of each element. The structure of the problem both at global level and at element level takes the form:

$$
\left(\cup_{e}\left[\begin{array}{ll}
\mathbf{A}^{e} & \mathbf{B}^{e T} \\
\mathbf{B}^{e} & -\mathbf{S}^{e}
\end{array}\right]\right)\left\{\begin{array}{c}
\mathbf{d} \\
\mathbf{m}
\end{array}\right\}=\cup_{e}\left\{\begin{array}{c}
\mathbf{f}^{e} \\
\mathbf{g}^{e}-\mathbf{t}^{* e}
\end{array}\right\}
$$

where the index $e$ indicates the element number and $\cup$ denotes assembly. $\mathbf{A}^{e}$ is the standard stiffness matrix and $\mathbf{f}^{e}$ is the equivalent force vector.

The definition of the Lagrange multiplier field as an interpolation based on the value of the multiplier at the quadrature point allow us to evaluate the matrices $\mathbf{B}_{e}$ and $\mathbf{S}_{e}$ and the vectors $\mathbf{g}_{e}$ and $\mathbf{t}_{e}^{*}$ without explicitly calculating the interpolation at each element. For example, for the evaluation of the constraint matrix, $\mathbf{B}^{e}$, we will have

$$
\int_{\Gamma^{e}} \boldsymbol{\mu}^{h} \mathbf{u}^{h} d \Gamma=\int_{-1}^{1} \boldsymbol{\mu}^{e} \mathbf{N}^{e} \mathbf{d}^{e} J d \zeta=\sum_{i g=1}^{n_{g}} \boldsymbol{\mu}_{i g}^{e} \mathbf{N}_{i g} H_{i g} J_{i g} \mathbf{d}^{e}=\sum_{i g=1}^{n_{g}} \boldsymbol{\mu}_{i g} \mathbf{B}_{i g}^{e} \mathbf{d}^{e}
$$

Note that every value $\boldsymbol{\mu}_{i g}$ multiply a row of matrix $\mathbf{B}^{e} . \mathbf{N}_{i g}$ is the shape function matrix evaluated at the quadrature point $i g$ of the contour integral, $H_{i g}$ is the weight and $J_{i g}$ is the Jacobian of the transformation.

Following a similar procedure, we obtain the diagonal stabilization matrix where each $2 \times 2$ block in the diagonal is the weight of each quadrature point that defines the multiplier times the determinant of the transformation, that is

$$
\mathbf{S}_{i g, i g}^{e}=H_{i g} J_{i g} I_{2 x 2} h^{e}
$$


Similarly, the values for point $i g$ on the right side of equation (23) are calculated as

$$
\mathbf{g}_{i g}^{e}=H_{i g} J_{i g} \mathbf{g}_{i g} \quad \mathbf{t}^{* e}{ }_{i g}=H_{i g} J_{i g} \boldsymbol{T}_{i g}^{*}
$$

where $\mathbf{g}_{i g}$ and $\boldsymbol{T}_{i g}^{*}$ denote variables evaluated at the quadrature point.

Since the Lagrange multipliers are decoupled and the element stabilizing matrix $\mathbf{S}^{e}$ is diagonal, it is very easy to condense the Lagrange multipliers from equation (23) at each element directly leading to a system of equations without the degrees of freedom of the multipliers:

$$
\left(\cup_{e}\left[\mathbf{A}^{e}+\mathbf{B}^{e T} \mathbf{S}^{e-1} \mathbf{B}^{e}\right]\right)\{\mathbf{d}\}=\cup_{e}\left\{\mathbf{f}^{e}+\mathbf{B}^{e T} \mathbf{S}^{e-1}\left(\mathbf{g}^{e}-\mathbf{t}^{* e}\right)\right\}
$$

\subsection{Evaluation of the first mesh}

The proposed methodology is based on the use of the solution $\boldsymbol{T}^{*}$ obtained in a previous (coarser) mesh of the refinement mesh sequence. Therefore, the question arises of how to solve the first mesh. The only constraint to select the Lagrange multiplier interpolation in the first mesh is to obtain a solvable system of equations. Note that once initiated, the overall process satisfies the convergence conditions.

We propose two methods, $M_{I}$ and $M_{I I}$, to solve the first mesh. Method $M_{I}$, considers the stabilization term using $\boldsymbol{T}^{*}=0$ in the first mesh. In this case the structure of the system matrix is unchanged. The second alternative, method $M_{I I}$, consists in defining a multiplier field such that the number of constraint equations (i.e. the number of multipliers) is lower than or equal to the number of degrees of freedom affected by the constraints (i.e. degrees of freedom of the intersected elements). To do this we choose the so-called naive approach, i.e. a piecewise linear interpolation with a degree of freedom at each intersection point of the boundary with the element edges. In this case, the matrix and vectors derived from the stabilization term in the first mesh are not taken into account.

\section{Numerical examples}

The proposed technique has been used to solve linear elasticity problems with exact solution in order to check the convergence of the proposed stabilization method. The finite element stresses directly calculated from the displacement interpolation have been used in the examples as stress stabilization term $\boldsymbol{T}^{*}$. In the case of $\mathcal{Q}_{4}$ elements the error of the Lagrange multipliers 
is much higher than the error in displacement field. An improved definition of the multipliers is defined based on the interpretation of the multipliers as the traction on the boundary. The definition is motivated by the observation that the nodal reaction forces of the Lagrange multipliers $\mathbf{r}=\mathbf{B}^{T} \mathbf{m}$, and the nodal reaction forces of the exact traction are very similar. We can define the boundary tractions as $\mathbf{p}^{h}=\mathbf{N}_{j} \mathbf{p}_{j}$ where the nodal value of the traction $\mathbf{p}_{j}$ is the reaction at node $j$ divided by its associated length over the boundary:

$$
\mathbf{p}_{j}=\frac{\mathbf{r}_{j}}{A_{j}}, \text { where } \quad A_{j}=\int_{\Gamma_{\mathrm{D}}} N_{j} d \Gamma
$$

We use a parameter $\kappa$ to define the penalty constant as $k=1 / \kappa E$, where $E$ is the Young modulus. The factor $1 / E$ introduced in the stabilization term $s(\cdot, \cdot)$ helps to make the equation dimensionally consistent.

\subsection{Example 1: Cubic polynomial displacements in a polygonal domain}

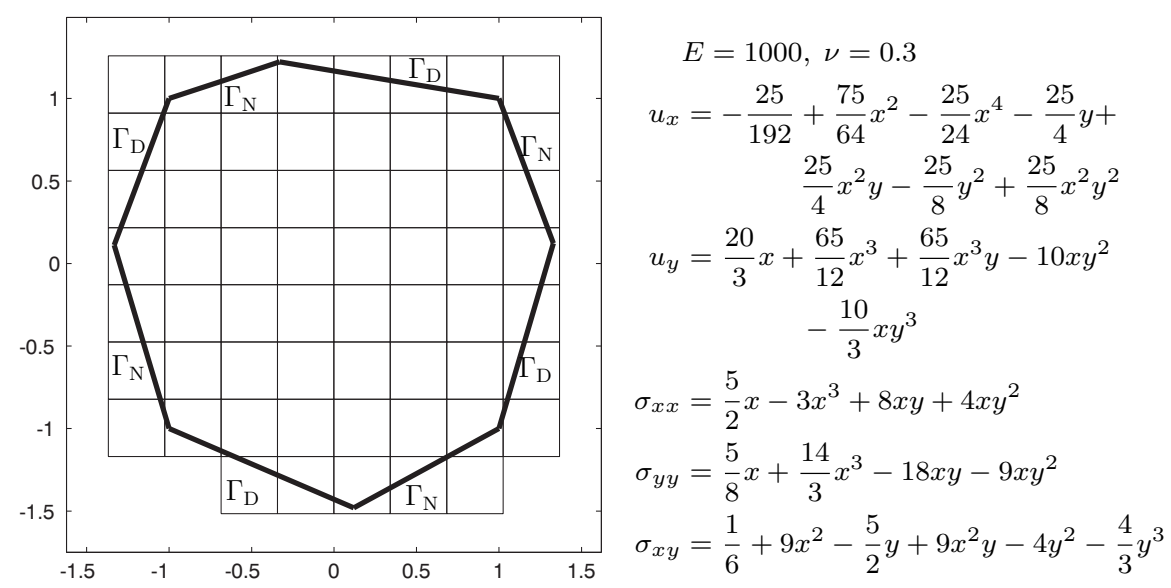

Figure 3: Initial mesh of the polygonal domain problem and exact solution.

The first example corresponds to the polygonal domain of boundary $\Gamma$ represented with thick lines in Figure (3) which has been embedded into a Cartesian uniform grid. Dirichlet and Neumann boundary conditions have been applied as shown in the figure. The right hand side of the figure shows the Young's modulus $E$ and Poisson's ratio $\nu$ of the material together with the expressions for the exact displacement and stress fields. Note that $\nabla \cdot \sigma=\mathbf{0}$, 


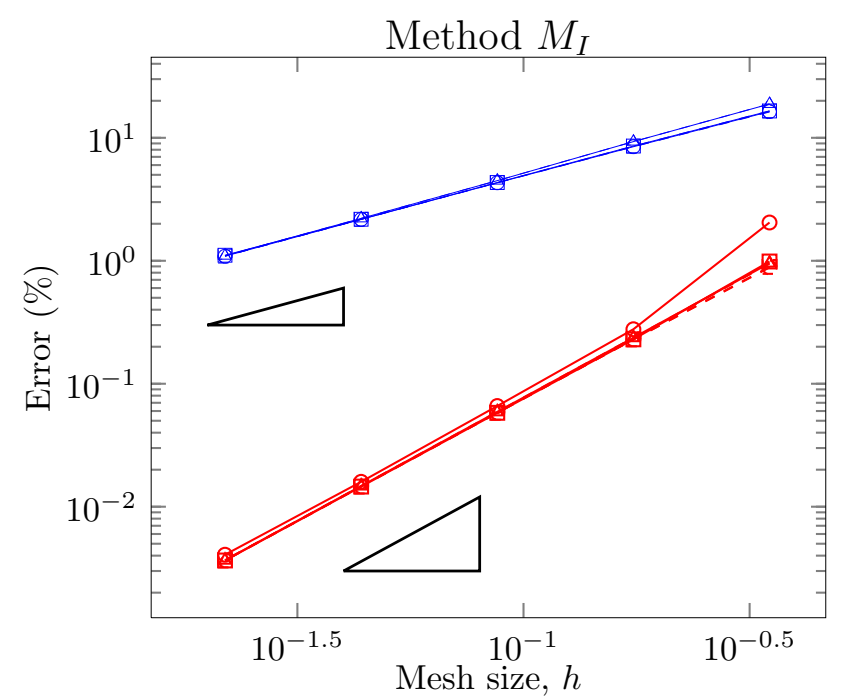

$$
\begin{aligned}
& \neg \mathcal{Q}_{4}, \kappa=10 \quad \square \mathcal{Q}_{4}, \kappa=100 \quad \triangle \mathcal{Q}_{4}, \kappa=1000 \\
& \multimap \mathcal{Q}_{8}, \kappa=20 \quad \square \mathcal{Q}_{8}, \kappa=100 \quad \triangle \mathcal{Q}_{8}, \kappa=1000 \\
& \text { - }-\mathcal{Q}_{4}, \kappa=10, \boldsymbol{T}_{\mathrm{eX}}^{*}-{ }^{\prime-} \mathcal{Q}_{4}, \kappa=100, \boldsymbol{T}_{\mathrm{ex}}^{*}+-\mathcal{Q}_{4}, \kappa=1000, \boldsymbol{T}_{\mathrm{ex}}^{*}
\end{aligned}
$$

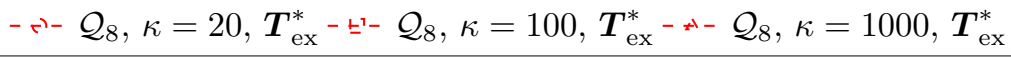

Figure 4: Problem 1. Discretization error in energy norm for $\mathcal{Q}_{4}$ and $\mathcal{Q}_{8}$ elements. Comparison of method $M_{I}$ with $\boldsymbol{T}^{*}=0$ and exact tractions for the initial mesh. The triangles show the optimal convergence, 1 for linear elements and 2 for quadratic elements.

therefore body loads $\mathbf{b}$ are zero in this case. The mesh shown in Figure 3 is the first one of a sequence of meshes generated by uniform refinement obtained by splitting each element into 4 new elements.

The exact discretization error in energy norm of the finite element solution has been evaluated for each of the meshes, considering different values of the penalty parameter $\kappa$. Figure (4) show the results for method $M_{I}$ and figure (5) for method $M_{I I}$. The curve show stable results for a wide range of values of $\kappa$. We can observe that the optimal convergence is achieved for linear and quadratic elements and both methods. The results show that the proposed technique produces convergence rates very close to the theoretical expected value for a wide rage of values of $k$.

To evaluate the influence of the solution projected from the first mesh in the error of the method, we solved the first mesh with the stabilized formulation using the exact traction in the field $\boldsymbol{T}^{*}$. The results are shown in figure (4). We see that the results obtained are very similar for a sufficiently 


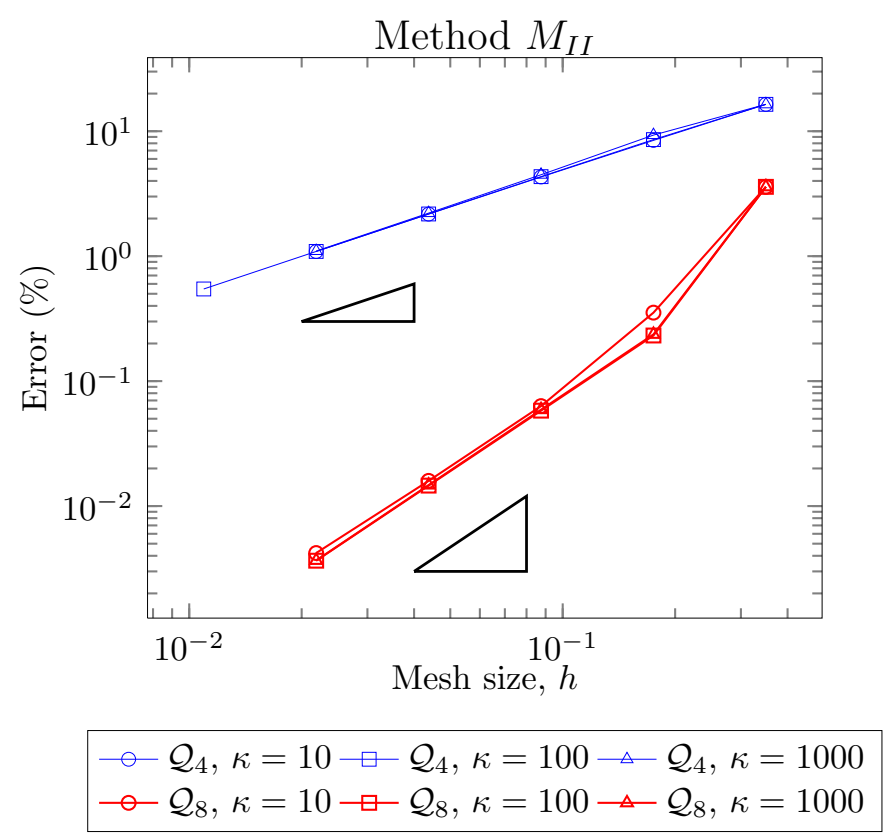

Figure 5: Problem 1. Discretization error in energy norm for $\mathcal{Q}_{4}$ and $\mathcal{Q}_{8}$ elements for method $M_{I I}$. The triangles show the optimal convergence, 1 for linear elements and 2 for quadratic elements. 
large $\kappa$. This indicates that the accuracy and convergence rates of the results obtained with the proposed methodology do not seem to be affected by the solution of the initial mesh in a wide range of values of $\kappa$. Of course, for very small values of $\kappa$ the stabilization term becomes the most important term in the energy, and the choice of $\boldsymbol{T}^{*}=0$ for the first mesh makes the solution to have a high error. This could also affect the convergence of the method. However another choice of the field $\boldsymbol{T}^{*}$ based on recovered stresses could improve this behavior.

The convergence of the Lagrange multiplier field is analyzed considering method $M_{I}$ in tables (1) and (2). These tables represent, for linear and quadratic elements, the error percentage of the multipliers in $L^{2}$ norm, and the error of the Lagrange multipliers and the stabilization term measured in the mesh dependent norm. The results are shown for a penalty parameter $\kappa=100$. The expected convergence [39] of Lagrange multipliers is $p$ in the $L^{2}$ norm and $p+\frac{1}{2}$ in the mesh dependent norm. The theoretical convergence of the stabilization term is also $p+\frac{1}{2}$. We see that these values are achieved in practice.

\begin{tabular}{|c|c|c|c|}
\hline Mesh & $\%\left\|\boldsymbol{\lambda}-\mathbf{p}^{h}\right\|_{L^{2}}$ & $\left\|\boldsymbol{\lambda}-\mathbf{p}^{h}\right\|_{\mathscr{M}^{h}}$ & $\left\|\boldsymbol{\lambda}-\boldsymbol{T}^{*}\right\|_{\mathscr{M}^{h}}$ \\
\hline 1 & $5.3292(-)$ & $435.27(-)$ & $1653.4(-)$ \\
\hline 2 & $2.9724(0.842)$ & $159.41(1.449)$ & $664.99(1.314)$ \\
\hline 3 & $1.2741(1.222)$ & $52.935(1.590)$ & $240.68(1.466)$ \\
\hline 4 & $0.44190(1.528)$ & $13.629(1.957)$ & $89.651(1.425)$ \\
\hline 5 & $0.23395(0.917)$ & $4.9035(1.475)$ & $31.279(1.519)$ \\
\hline
\end{tabular}

Table 1: Problem 1. Error of the tractions and convergence rate (in brackets, theoretically expected values $1,1.5$ and 1.5 ) for $\mathcal{Q}_{4}$ elements using method $M_{I}$.

\begin{tabular}{|c|c|c|c|}
\hline Mesh & $\%\left\|\boldsymbol{\lambda}-\boldsymbol{\lambda}^{h}\right\|_{L^{2}}$ & $\left\|\boldsymbol{\lambda}-\boldsymbol{\lambda}^{h}\right\|_{\mathscr{M}^{h}}$ & $\left\|\boldsymbol{\lambda}-\boldsymbol{T}^{*}\right\|_{\mathscr{M}^{h}}$ \\
\hline 1 & $18.554(-)$ & $1048.6(-)$ & $161.41(-)$ \\
\hline 2 & $1.5460(3.585)$ & $81.298(3.689)$ & $16.389(3.300)$ \\
\hline 3 & $0.38779(1.995)$ & $14.463(2.491)$ & $2.7832(2.558)$ \\
\hline 4 & $0.10063(1.946)$ & $2.6921(2.426)$ & $0.51402(2.437)$ \\
\hline 5 & $2.4427 \cdot 10^{-2}(2.042)$ & $0.47707(2.496)$ & $9.1564 \cdot 10^{-2}(2.489)$ \\
\hline
\end{tabular}

Table 2: Problem 1. Error of the tractions and convergence rate (in brackets, theoretically expected values $2,2.5$ and 2.5) for $\mathcal{Q}_{8}$ elements using method $M_{I}$. 


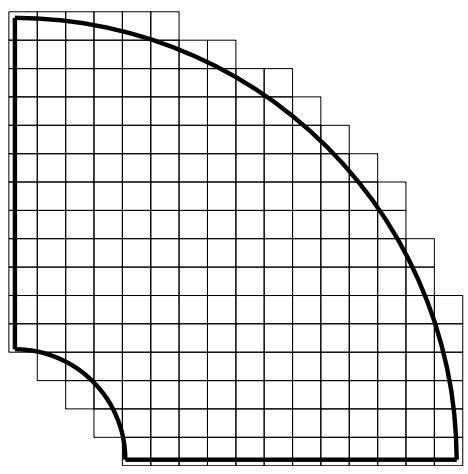

Figure 6: Initial mesh of the hollow cylinder problem.

The second example, shown in figure (6), corresponds to the analysis of a hollow cylinder subjected to internal pressure. This figure shows the first mesh of the sequence of uniformly refined meshes obtained by element splitting. The numerical analysis considered in the previous were also used in this case. Figures (7) and (8) show the relative discretization error in energy norm for linear and quadratic elements using methods $M_{I}$ and $M_{I I}$, respectively. Tables (3) and (4) show the error in the tractions using $\kappa=100$. The results presented in these figures and tables are similar to those presented in the first example. Note that again method $M_{I}$ provides results similar to method $M_{I I}$. Therefore, we suggest the use of method $M_{I}$ which does not require any special treatment of the first mesh.

\begin{tabular}{|c|c|c|c|}
\hline Mesh & $\%\left\|\boldsymbol{\lambda}-\mathbf{p}^{h}\right\|_{L^{2}}$ & $\left\|\boldsymbol{\lambda}-\mathbf{p}^{h}\right\|_{\mathscr{M}^{h}}$ & $\left\|\boldsymbol{\lambda}-\boldsymbol{T}^{*}\right\|_{\mathscr{M}^{h}}$ \\
\hline 1 & $3.1200(-)$ & $0.13015(-)$ & $0.26577(-)$ \\
\hline 2 & $1.4397(1.116)$ & $4.0141 \cdot 10^{-2}(1.697)$ & $8.5765 \cdot 10^{-2}(1.632)$ \\
\hline 3 & $0.63127(1.189)$ & $1.0730 \cdot 10^{-2}(1.903)$ & $3.1371 \cdot 10^{-2}(1.451)$ \\
\hline 4 & $0.20679(1.610)$ & $2.4429 \cdot 10^{-3}(2.135)$ & $1.1612 \cdot 10^{-2}(1.434)$ \\
\hline 5 & $7.8993 \cdot 10^{-2}(1.388)$ & $5.7586 \cdot 10^{-4}(2.085)$ & $4.1385 \cdot 10^{-3}(1.488)$ \\
\hline
\end{tabular}

Table 3: Problem 2. Error of the tractions and convergence rate (in brackets, theoretically expected values are $1,1.5$ and 1.5) for $\mathcal{Q}_{4}$ elements using method $M_{I}$.

In this example, we also solved the problem using an $h$-adaptive mesh refinement. Multipoint constraints are used to impose the continuity of the 


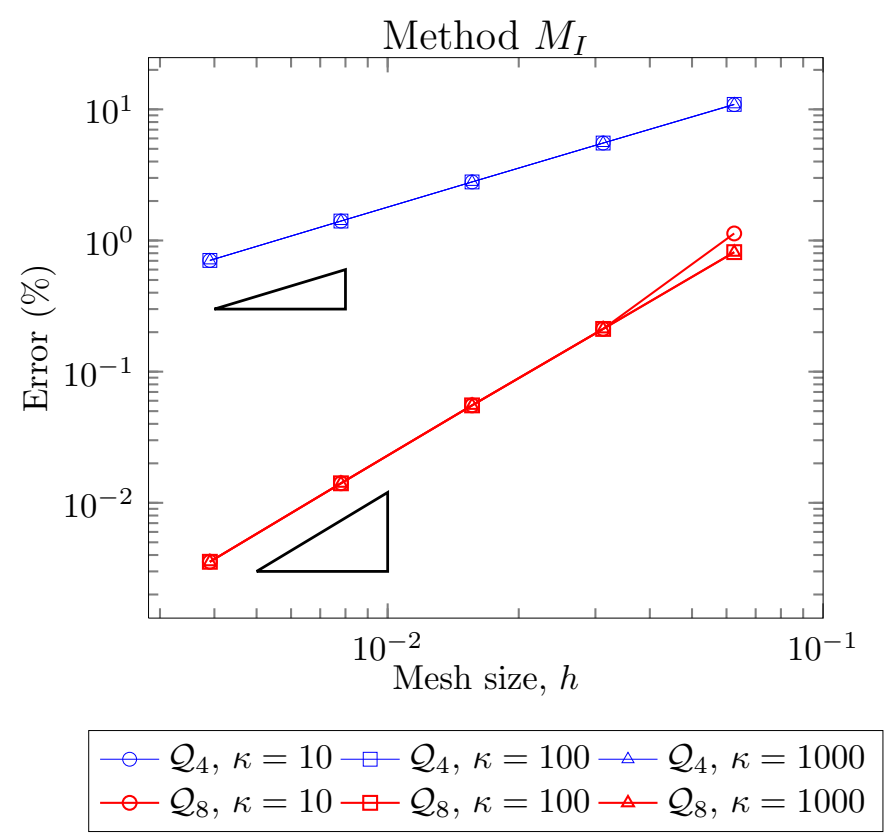

Figure 7: Problem 2. Discretization error in energy norm for $\mathcal{Q}_{4}$ and $\mathcal{Q}_{8}$ elements for method $M_{I}$. The triangles show the optimal convergence, 1 for linear elements and 2 for quadratic elements. 


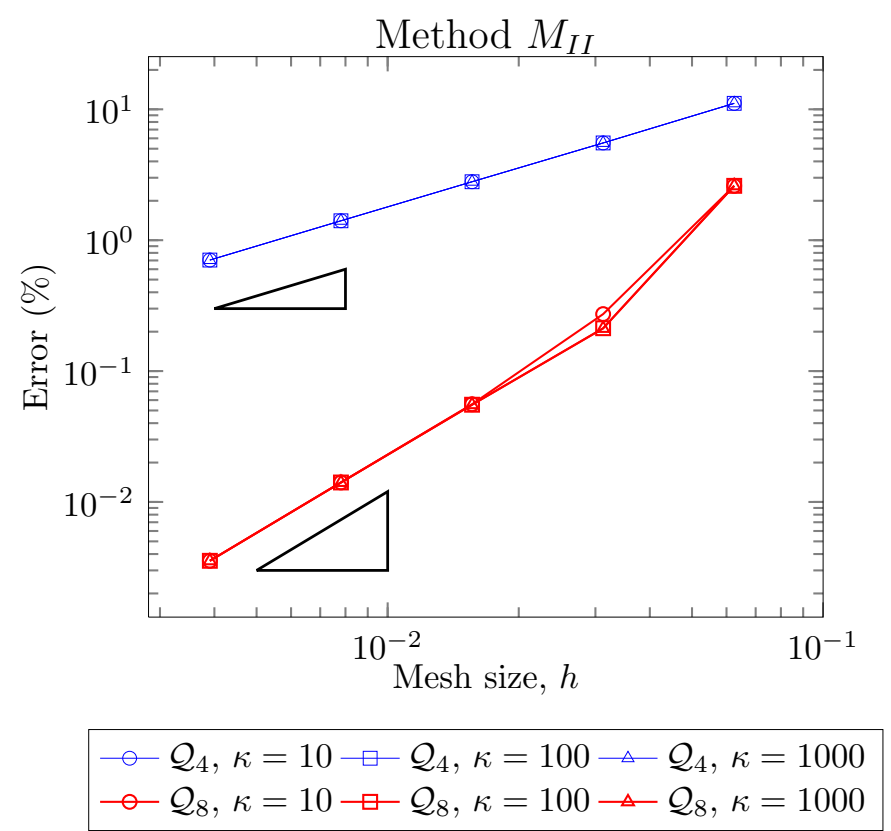

Figure 8: Problem 2. Discretization error in energy norm for $\mathcal{Q}_{4}$ and $\mathcal{Q}_{8}$ elements for method $M_{I I}$. The triangles show the optimal convergence, 1 for linear elements and 2 for quadratic elements. 


\begin{tabular}{|c|c|c|c|}
\hline Mesh & $\%\left\|\boldsymbol{\lambda}-\boldsymbol{\lambda}^{h}\right\|_{L^{2}}$ & $\left\|\boldsymbol{\lambda}-\boldsymbol{\lambda}^{h}\right\|_{\mathscr{M}^{h}}$ & $\left\|\boldsymbol{\lambda}-\boldsymbol{T}^{*}\right\|_{\mathscr{M}^{h}}$ \\
\hline 1 & $1.6026(-)$ & $6.6828 \cdot 10^{-2}(-)$ & $7.7181 \cdot 10^{-2}(-)$ \\
\hline 2 & $0.20229(2.986)$ & $6.1750 \cdot 10^{-3}(3.436)$ & $8.9338 \cdot 10^{-3}(3.111)$ \\
\hline 3 & $3.9818 \cdot 10^{-2}(2.345)$ & $8.6503 \cdot 10^{-4}(2.835)$ & $7.9124 \cdot 10^{-4}(3.497)$ \\
\hline 4 & $1.1425 \cdot 10^{-2}(1.801)$ & $1.7420 \cdot 10^{-4}(2.312)$ & $1.7010 \cdot 10^{-4}(2.218)$ \\
\hline 5 & $3.3726 \cdot 10^{-3}(1.760)$ & $3.3331 \cdot 10^{-5}(2.386)$ & $6.2112 \cdot 10^{-5}(1.453)$ \\
\hline 6 & $7.4312 \cdot 10^{-4}(2.182)$ & $5.6048 \cdot 10^{-6}(2.572)$ & $5.0478 \cdot 10^{-6}(3.621)$ \\
\hline
\end{tabular}

Table 4: Problem 2. Error of the tractions and convergence rate (in brackets, theoretically expected values are 2, 2.5 and 2.5) for $\mathcal{Q}_{8}$ elements using method $M_{I}$.

displacement field in the hanging-nodes. The first four meshes of the sequence are plotted in figure (9). The percentage of error in energy norm is plotted in figure (10) as a function of the number of degrees of freedom of the mesh. The value of $\kappa=100$ was used. A comparison with the results of uniform refinement is shown in the same figure. Note that in this case, the optimal convergence rate is 0.5 for linear elements and 1 for quadratic elements because the error is plotted versus the number of degrees of freedom instead of the element size. As can be observed, to obtain the same level of error the $h$-adapted mesh needs lower number of degrees of freedom.

\section{Conclusions}

A new a stabilized method to impose Dirichlet boundary conditions using the Lagrange multipliers method in FE meshes not conforming to the geometry of the domain has been presented. The stabilization term penalizes the difference between the Lagrange multiplier field associated to a mesh and the finite element stress on the Dirichlet boundary obtained in a coarser mesh. This characteristic of our method simplifies the formulation and implementation as the stabilizing term $\boldsymbol{T}^{*}$ does not depend on the current mesh.

It has been demonstrated that a sufficiently small value of the penalty parameter must provide the optimal convergence rate of the discretization error in energy norm, 1 for linear elements and 2 for quadratic elements, as a function of the element size. The numerical results in Section 7 show that the optimal convergence rate of the finite element error is obtained in both cases for a wide range of values of the penalty parameter. The errors obtained with different values of the penalty parameter $k$ and different techniques to obtain the solution of the first analysis mesh, whose results are required to 

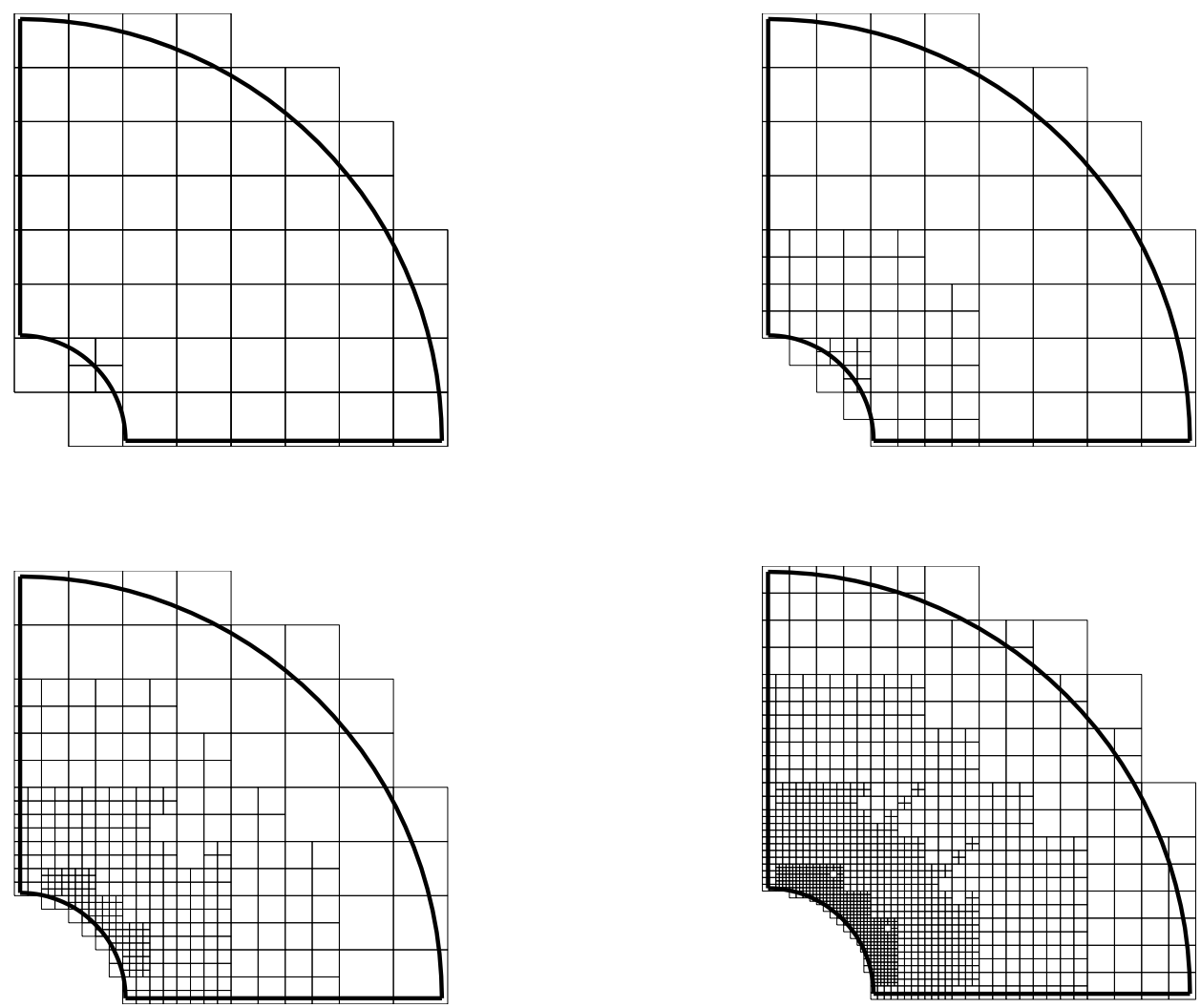

Figure 9: Sequence of meshes obtained by $h$-adaptive refinement using $\mathcal{Q}_{4}$ elements, $\operatorname{method} M_{I}$ and $\kappa=100$. 


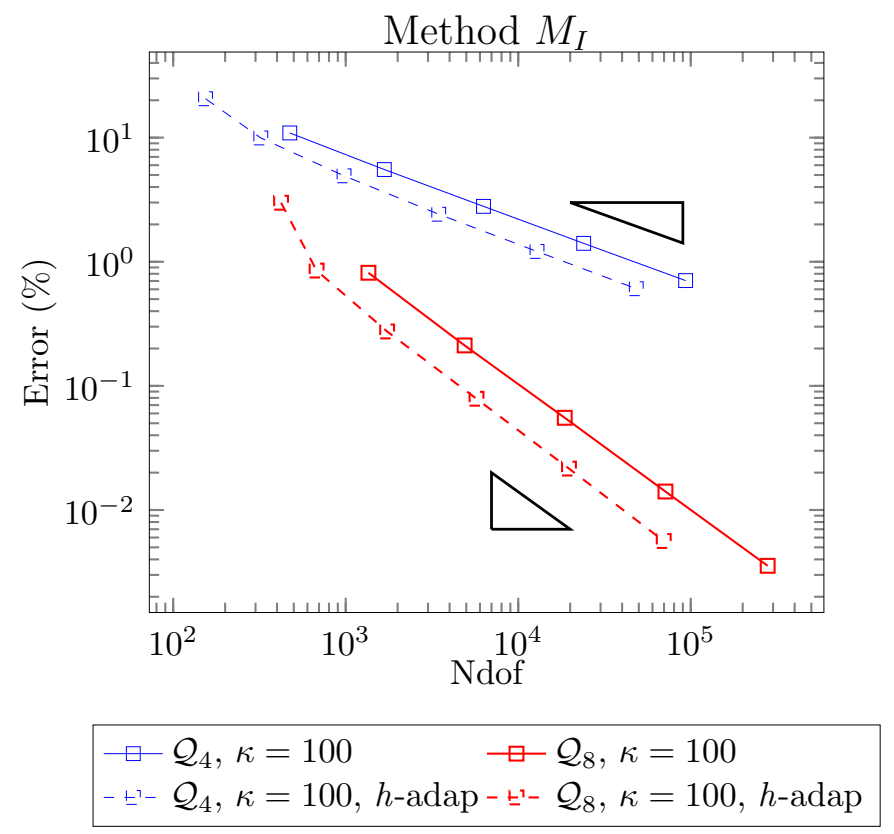

Figure 10: Problem 2. Discretization error (\%) in energy norm for $\mathcal{Q}_{4}$ and $\mathcal{Q}_{8}$ elements for method $M_{I}$ and $\kappa=100$. Comparison of uniform refinement and $h$-adapted mesh. The triangles show the optimal convergence, 0.5 for linear elements and 1 for quadratic elements. 
start the process, remain practically unchanged. The numerical analysis show that even the use of $\boldsymbol{T}^{*}=0$ for the first mesh is a reasonable alternative. An additional advantage of the proposed method is that the Lagrange multipliers can be eliminated from the system of equations of each element, thus, the overall size of the system of equations is not increased by use of the Lagrange multipliers technique.

\section{Acknowledgements}

The authors acknowledge the financial support received from the research project DPI2010-20542 of the Ministerio de Economía y Competitividad. Also it is appreciated the financial support of the FPU program (AP200801086) of the Universitat Politècnica de Valencia and the Generalitat Valenciana (PROMETEO/2012/023). The authors are also grateful for support support of the Framework Program 7 Initial Training Network Funding under grant number 289361 'Integrating Numerical Simulation and Geometric Design Technology (INSIST).”

[1] Cottrell JA, Hughes TJR, Bazilevs Y. Isogeometric Analysis: Toward Integration of CAD and FEA. 1st edn., Wiley, 2009.

[2] Moës N, Dolbow J, Belytschko T. A finite element method for crack growth without remeshing. International Journal for Numerical Methods in Engineering 1999; 46(1):131-150.

[3] Sukumar N, Prévost JH. Modeling quasi-static crack growth with the extended finite element method. \{Part I\}: Computer implementation. International Journal of Solids and Structures 2003; 40(26):7513-7537.

[4] Strouboulis T, Copps K, Babuška I. The generalized finite element method. Computer Methods in Applied Mechanics and Engineering May 2001; 190(32-33):4081-4193.

[5] Strouboulis T, Zhang L, Babuscaronka I. Assessment of the cost and accuracy of the generalized FEM. International Journal for Numerical Methods in Engineering 2007; 69(2):250-283.

[6] Heikkola E, Kunzetsov YA, Lipnikov KN. Fictitious domain methods for the numerical solution of three-dimensional acoustic scattering problems. Journal of Computational Acoustics 1999; 7(3):161-193. 
[7] DeRose Jr G, Díaz A. Solving three-dimensional layout optimization problems using fixed scale wavelets. Computational Mechanics 2000; 25:274-285.

[8] Ramière I, Angot P, Belliard M. Fictitious domain methods to solve convection-diffusion problems with general boundary conditions. Proc. in the 17th Computational Fluid Dynamics Conference AIAA, 2005.

[9] Parussini L, Pedirola V. Fictitious Domain approach with hp-finite element approximation for incompressible fluid flow. Journal of Computational Physics 2009; 228:3891-3910.

[10] Bishop J. Rapid stress analysis of geometrically complex domains using implicit meshing. Computational Mechanics 2003; 30:460-478.

[11] Zhang L, Gerstenberger A, Wang X, Liu W. Immersed finite element method. Computer Methods in Applied Mechanics and Engineering 2004; 193:2051-2067.

[12] Ye T, Mittal R, Udaykumar H, Shyyy W. Accurate Cartesian grid method for viscous incompressible flows with complex immersed boundaries. Journal of Computational Physics 1999; 156:209-240.

[13] Roma A, Peskin C, Bergery M. An adaptive version of the immersed boundary method. Journal of Computational Physics 1999; 153:509534 .

[14] García-Ruíz M, Steven G. Fixed grid finite elements in elasticity problems. Engineering Computations 1999; 16:145-164.

[15] Daneshmand F, Kazemzadeh-Parsi M. Static and dynamic analysis of 2D and 3D elastic solids using the modified FGFEM. Finite Elements in Analysis and Design 2009; 228:3891-3910.

[16] Bordas SPA, Rabczuk T, Ródenas J, Kerfriden P, Moumnassi M, Belouettar S. Recent advances towards reducing the meshing and re-meshing burden in computational sciences. Computational Technology Reviews: 2, Topping B, Adam J, Pallarés F, Bru R, Romero M (eds.). SaxeCoburg Publications: Stirlingshire, 2010. 
[17] Babuska I. The finite element method with Lagrangian multipliers. $\mathrm{Nu}$ merische Mathematik 1973; 20(3):179-192.

[18] Pitkaranta J. A conforming finite element method with Lagrange multipliers for the biharmonic problem. ESAIM: Mathematical Modelling and Numerical Analysis - Modélisation Mathématique et Analyse Numérique 1980; 14(3):309-324.

[19] Brezzi F, Fortin M. Mixed and hybrid finite element methods. SpringerVerlag New York, Inc.: New York, NY, USA, 1991.

[20] Béchet E, Moës N, Wohlmuth B. A stable Lagrange multiplier space for stiff interface conditions within the extended finite element method. International Journal for Numerical Methods in Engineering 2009; 78(8):931-954.

[21] Hautefeuille M, Annavarapu C, Dolbow JE. Robust imposition of Dirichlet boundary conditions on embedded surfaces. International Journal for Numerical Methods in Engineering 2012; 90(1):40-64.

[22] Barbosa HJ, Hughes TJ. Boundary Lagrange multipliers in finite element methods: Error analysis in natural norms. Numerische Mathematik 1992; 62:1-15.

[23] Bochev PB, Dohrmann CR, Gunzburger MD. Stabilization of low-order mixed finite elements for the Stokes equations. SIAM J. Numer. Anal. Jan 2006; 44(1):82-101.

[24] Hansbo A, Hansbo P. An unfitted finite element method based on Nitsche method for elliptic interface problems. Computer Methods in Applied Mechanics and Engineering 2002; 191:5537 - 5552.

[25] Haslinger J, Renard Y. A new fictitious domain approach inspired by the extended finite element method. SIAM Journal on Numerical Analysis Mar 2009; 47(2):1474-1499.

[26] Liu F, Borja RI. Stabilized low-order finite elements for frictional contact with the extended finite element method. Computer Methods in Applied Mechanics and Engineering 2010; 199(37-40):2456 - 2471. 
[27] Burman E, Hansbo P. Fictitious domain finite element methods using cut elements: I. A stabilized Lagrange multiplier method. Computer Methods in Applied Mechanics and Engineering 2010; 199:2680 - 2686.

[28] Burman E, Hansbo P. Fictitious domain finite element methods using cut elements: II. A stabilized Nitsche method. Applied Numerical Mathematics 2012; 62(4):328 - 341.

[29] Brezzi F. On the existence, uniqueness and approximation of saddlepoint problems arising from Lagrangian multipliers. ESAIM: Mathematical Modelling and Numerical Analysis - Modélisation Mathématique et Analyse Numérique 1974; 8(R2):129-151.

[30] Nadal E, Ródenas JJ, Albelda J, Tur M, Tarancón JE, Fuenmayor FJ. Efficient finite element methodology based on Cartesian grids: Application to structural shape optimization. Abstract and Applied Analysis $2013 ;: 1-19$.

[31] Stenberg R. On some techniques for approximating boundary conditions in the finite element method. Journal of Computational and Applied Mathematics 1995; 63:139-148.

[32] Burman E. Projection stabilisation of Lagrange multipliers for the imposition of constraints on interfaces and boundaries. arXiv:1203.4116, 2012.

[33] Barbosa HJC, Hughes TJR. Circumventing the Babuska-Brezzi condition in mixed finite element approximations of elliptic variational inequalities. Computer Methods in Applied Mechanics and Engineering Jun 1992; 97(2):193-210.

[34] Codina R, Baiges J. Approximate imposition of boundary conditions in immersed boundary methods. International Journal for Numerical Methods in Engineering 2009; 80(11):1379-1405.

[35] Nadal E, Bordas S, Ródenas J, Tarancón J, Tur M. Accurate stress recovery for the two-dimensional fixed grid finite element method. Proceedings of the Tenth International Conference on Computational Structures Technology, Topping B, Adam J, Pallarés F, Bru R, Romero M (eds.). Civil-Comp Press: Stirlingshire UK, 2010. 
[36] Ródenas JJ, Tur M, Fuenmayor FJ, Vercher A. Improvement of the superconvergent patch recovery technique by the use of constraint equations: the SPR-C technique. International Journal for Numerical Methods in Engineering 2007; 70(6):705-727.

[37] Ródenas JJ, González-Estrada OA, Tarancón JE, Fuenmayor FJ. A recovery-type error estimator for the extended finite element method based on singular+smooth stress field splitting. International Journal for Numerical Methods in Engineering 2008; 76(4):545-571.

[38] Sanders JD, Dolbow JE, Laursen TA. On methods for stabilizing constraints over enriched interfaces in elasticity. International Journal for Numerical Methods in Engineering 2009; 78(9):1009-1036.

[39] Wohlmuth BI. A comparison of dual Lagrange multiplier spaces for mortar finite element discretizations. Mathematical Modeling and Numerical Analysis 2002; 36(6):995 - 1012. 